\title{
An analytical approach to the unified solution of kinetic equations in the rarefied gas dynamics. II. Heat transfer problems
}

\author{
C. S. Scherer, J. F. Prolo Filho and L. B. Barichello*
}

\begin{abstract}
The ADO method, an analytical version of the discrete-ordinates method, is used here to solve a heat-transfer problem in a rarefied gas confined in a channel, as well as to solve a half-space problem in order to evaluate the temperature jump at the wall. This work is an extension of a previous work, devoted to flow problems, where the complete development of the solution, which is analytical in terms of the spatial variable, is presented in a way, such that, a wide class of kinetic models are considered, in an unified approach. A series of numerical results are showed and different simulations are used in order to establish a general comparative analysis based on this consistent set of results provided by the same methodology. In particular, numerical results for heat-flow profile, temperature and density perturbations are obtained for channels (walls), defined by different materials, on which different temperatures are imposed.
\end{abstract}

Keywords. Rarefied gas dynamics, Boltzmann equation, kinetic models, temperature jump, heat transfer, ADO method.

\section{Introduction}

The heat-transfer problem in a rarefied gas, confined in a channel defined by two parallel plates where different temperatures are imposed, has been studied intensively in the literature over the years [1] - [11], as well as, the temperature-jump problem [12]-[17]. Specially because of some modern technological applications, as micro-systems [18] - [22], these problems have been revisited, in the search for precise results provided by fast and reliable tools. In fact, for the micro-flows case, when significant rarefaction effects have to be taken into account, in the transition regime, the usual fluid dynamics approach does not seem to be appropriate. The gas flow has to be characterized by the distribution function of the gas particles, which can be obtained either from the solution of the Boltzmann equation (BE) or from kinetic model equations derived from the $\mathrm{BE}$ - where simplified integral collision operators are introduced [23] - [25].

In recent years, analytical solutions in terms of the spatial variable have been

\footnotetext{
*Corresponding author
} 
developed, for both of the above mentioned problems [8], [26] - [30], by the ADO method [31], which is an analytical version of the discrete-ordinates method [32]. In these listed works, different procedures were proposed to deal with kinetic equations, as the BGK [33] and the S model [34] equations. However, the ADO method has been also applied, along with an expansion technique, to derive solutions for the linearized Boltzmann equation $[10,35,36]$. The ADO method has been shown to be very accurate, fast and easy to implement and, in this sense, a class of problems has been solved by this approach. In fact, in a series of recent works, "unified solutions" for different problems, have been developed for some kinetic models [37]-[40].

In a very recent work [41], we were able to develop an unified approach, based on the ADO method, which solution includes a whole class of kinetic equations, more precisely, the BGK model [33], the S model [34], the Gross-Jackson (GJ) model [42] and the MRS model [43]. In that work, the ADO solution was able to represent in an unified approach the solution of a wide a class of flow problems for all the four kinetic equations.

In this work, we extend this idea, of developing an analytical solution which is valid for all four kinetic models, for describing heat transfer problems. In addition, we present original results for the GJ [42] and MRS [43] models. Our final goal is to establish the main procedures to apply the ADO method, which involves some analytical steps, to a (as much as possible) major class of problems, such that, its implementation will depend on just a few steps. Furthermore, based on the same methodology, a comparative analysis for a wide class of problems in rarefied gas dynamics, including the problems mentioned above, with results obtained from different kinetic equations and the LBE equation, may be established. Still, we introduce an analysis of the surface effects (accommodation coefficients), since in our formulation we allow different accommodation coefficients in each one of the surfaces.

In this way, we present the kinetic formulation of the problems to be solved in Sec. 2. In Sec. 3 an appropriate reformulation is presented, in order to make it possible the development of the discrete-ordinates approach detailed in Sec. 4. In Sec. 5 the ADO solution is used to evaluate physical quantities of interest. Computational aspects and numerical results are discussed in Sec. 6, and finally some concluding comments are given in Sec. 7 .

\section{Kinetic equations}

We start here from the non-linear Boltzmann equation written, in a general form [44], as

$$
\mathbf{v} \cdot \nabla_{r} f(\mathbf{r}, \mathbf{v})=J\left(f^{\prime}, f\right)
$$

where $f(\mathbf{r}, \mathbf{v})$ is the gas atom space and velocity distribution function $\left(f^{\prime}\right.$ and $f$ are associated with, respectively, before and after collisions distributions) and $J$ is the 
collision operator [44]. We write $f$, for the cases weakly far from the equilibrium, as

$$
f(\mathbf{r}, \mathbf{v})=f_{0}(\mathbf{v})[1+h(\mathbf{r}, \mathbf{v})]
$$

where $h$ is a perturbation to the absolute Maxwellian $f_{0}(\mathbf{v})$

$$
f_{0}(\mathbf{v})=n_{0}\left(\lambda_{0} / \pi\right)^{3 / 2} \mathrm{e}^{-\lambda_{0} v^{2}}, \quad \lambda_{0}=m_{0} /\left(2 k T_{0}\right) .
$$

Here $k$ is the Boltzmann constant, $T_{0}$ is a reference temperature, $m_{0}$ is the mass and $n_{0}$ is the equilibrium density of the gas. We substitute Eq. (2) into Eq. (1) and use, along with properties of the collision operator, some physical considerations [23], to obtain, for the dimensionless velocity variable

$$
\mathbf{c}=\mathbf{v}\left(m_{0} / 2 k T_{0}\right)^{1 / 2},
$$

the one-dimensional linearized equation written in terms of the perturbation function $h$, as $[44,45]$

$$
c_{x} \frac{\partial}{\partial \tau} h(\tau, \mathbf{c})+\varepsilon h(\tau, \mathbf{c})=\varepsilon \pi^{-3 / 2} \int_{-\infty}^{\infty} \int_{-\infty}^{\infty} \int_{-\infty}^{\infty} \mathrm{e}^{-c^{\prime 2}} h\left(\tau, \mathbf{c}^{\prime}\right) F\left(\mathbf{c}^{\prime}: \mathbf{c}\right) \mathrm{d} c_{x}^{\prime} \mathrm{d} c_{y}^{\prime} \mathrm{d} c_{z}^{\prime} .
$$

We consider here the dimensionless variable $\tau=x / l$ written in terms of a mean-free path $l$, which, at this point, we leave arbitrary, and

$$
\varepsilon=\sigma_{0}^{2} n_{0} \pi^{1 / 2} l
$$

where $\sigma_{0}$ is the collision diameter of the gas particles (in the rigid-sphere approximation).

Still, in regard to Eq. (5), the scattering kernel is written, in this work, as

$F\left(\mathbf{c}^{\prime}: \mathbf{c}\right)=1+2\left(\mathbf{c}^{\prime} \cdot \mathbf{c}\right)+(2 / 3)\left(c^{\prime 2}-3 / 2\right)\left(c^{2}-3 / 2\right)+\beta M\left(\mathbf{c}^{\prime}: \mathbf{c}\right)+\varpi N\left(\mathbf{c}^{\prime}: \mathbf{c}\right)$,

with

$$
M\left(\mathbf{c}^{\prime}: \mathbf{c}\right)=(4 / 5)\left(\mathbf{c}^{\prime} \cdot \mathbf{c}\right)\left(c^{2}-5 / 2\right)\left(c^{2}-5 / 2\right)
$$

and

$$
N\left(\mathbf{c}^{\prime}: \mathbf{c}\right)=2\left[\left(\mathbf{c}^{\prime} \cdot \mathbf{c}\right)^{2}-(1 / 3) c^{\prime 2} c^{2}\right]
$$

such that, for appropriate choices of the parameters $\beta$ and $\varpi$, following previous references $[26,43,46]$, we can define four kinetic equations. In addition, as showed by Barichello and Siewert [45], depending on the kinetic model, the parameter $\varepsilon$ will also assume different values, when defined in terms of a mean-free-path evaluated in terms of viscosity $\left(\varepsilon_{p}\right)$ or thermal-conductivity $\left(\varepsilon_{t}\right)$.

In this way, to be clear, we complete the definition of Eqs. (5) and (7) as follows $[26,43,45,46]$

\section{- the BGK model}

$$
\beta=\varpi=0, \quad \varepsilon_{t}=\varepsilon_{p}=1, \quad \varepsilon_{p} / \varepsilon_{t}=1
$$


- the $\mathrm{S}$ model

$$
\beta=1 / 3, \quad \varpi=0, \quad \varepsilon_{t}=3 / 2, \quad \varepsilon_{p}=1, \quad \varepsilon_{p} / \varepsilon_{t}=2 / 3
$$

- the Gross-Jackson (GJ) model

$$
\beta=5 / 9, \quad \varpi=1 / 3, \quad \varepsilon_{t}=9 / 4, \quad \varepsilon_{p}=3 / 2, \quad \varepsilon_{p} / \varepsilon_{t}=2 / 3
$$

- the MRS model

$$
\begin{gathered}
\beta=1-(16 / 15) 2^{1 / 2}, \quad \varpi=1-(8 / 5) 2^{1 / 2}, \quad \varepsilon_{t}=(15 / 32) 2^{1 / 2}, \\
\varepsilon_{p}=(5 / 16) 2^{1 / 2}, \quad \varepsilon_{p} / \varepsilon_{t}=2 / 3 .
\end{gathered}
$$

To add a few more comments in regard to the models cited, we note that, from Eqs. (8) to (11), it is easy to check a known difference between the BGK model and the other ones (although all of them are constant collision frequency models), in regard to the poor evaluation of the Prandtl number $\left(\varepsilon_{p} / \varepsilon_{t}=1\right)$. On the other hand, we emphasize that according to the derivation proposed in Ref. [45] and extended in Ref. [47], the BGK and the S models correspond to a two terms expansion in a synthetic approximation of the exact kernel proposed by Pekeris and Alterman [48] for rigid-spheres approximation, while the GJ and the MRS models represent a three therms expansion, as showed in Ref. [43].

Still, in regard to Eqs. (5) and (7), based on the three components of the velocity vector, $\left(c_{x}, c_{y}, c_{z}\right)$, with magnitude $c$, it is clear that

$$
\mathbf{c}^{\prime} \cdot \mathbf{c}=c_{x}^{\prime} c_{x}+c_{y}^{\prime} c_{y}+c_{z}^{\prime} c_{z} .
$$

In terms of the perturbation distribution $h$, some quantities of interest we want to evaluate in this work, are, respectively, the perturbation of density and temperature [44],

$$
\begin{gathered}
N(\tau)=\pi^{-3 / 2} \int_{-\infty}^{\infty} \int_{-\infty}^{\infty} \int_{-\infty}^{\infty} \mathrm{e}^{-c^{2}} h(\tau, \mathbf{c}) \mathrm{d} c_{x} \mathrm{~d} c_{y} \mathrm{~d} c_{z}, \\
T(\tau)=\frac{2}{3} \pi^{-3 / 2} \int_{-\infty}^{\infty} \int_{-\infty}^{\infty} \int_{-\infty}^{\infty} \mathrm{e}^{-c^{2}}\left(c^{2}-3 / 2\right) h(\tau, \mathbf{c}) \mathrm{d} c_{x} \mathrm{~d} c_{y} \mathrm{~d} c_{z}
\end{gathered}
$$

and the heat flow [11]

$$
Q_{x}=\pi^{-3 / 2} \int_{-\infty}^{\infty} \int_{-\infty}^{\infty} \int_{-\infty}^{\infty} \mathrm{e}^{-c^{2}}\left(c^{2}-5 / 2\right) h(\tau, \mathbf{c}) c_{x} \mathrm{~d} c_{x} \mathrm{~d} c_{y} \mathrm{~d} c_{z} .
$$

We note that, if we multiply Eq. (5) by $c^{k} \mathrm{e}^{-c^{2}}$ for $k=0,2$, and we integrate over all $c_{x}, c_{y}, c_{z}$, we show that the heat flow is independent of the spatial variable $[44]$.

At this point, to complete the definition of the problems to be solved, we have to supplement Eq. (5) with boundary conditions. In what follows we write them explicitly, noting that for the heat-transfer problem, we consider a channel of thickness $2 a$, such that, in Eq. (5), $\tau \in[-a, a]$; for the half-space problem which defines the temperature jump, we consider $\tau \in[0, \infty)$. 


\subsection{The heat-transfer problem}

For this plane channel problem, we consider the walls with (dimensionless) temperatures $T_{\omega 1}$ and $T_{\omega 2}$, respectively, on $\tau=-a$ and $\tau=a$. Repeating the linearization process introduced in Eq. (2), for the boundary conditions, we obtain [44] the linearized boundary conditions, for the case of Maxwell law, written for $c_{x}>0$, as

$$
\begin{aligned}
h\left(-a, c_{x}, c_{y}, c_{z}\right) & =\alpha_{1} \delta_{1}\left(c^{2}-2\right)+\left(1-\alpha_{1}\right) h\left(-a,-c_{x}, c_{y}, c_{z}\right) \\
& +\frac{2 \alpha_{1}}{\pi} \int_{-\infty}^{\infty} \int_{-\infty}^{\infty} \int_{0}^{\infty} \mathrm{e}^{-c^{\prime 2}} h\left(-a,-c_{x}^{\prime}, c_{y}^{\prime}, c_{z}^{\prime}\right) c_{x}^{\prime} \mathrm{d} c_{x}^{\prime} \mathrm{d} c_{y}^{\prime} \mathrm{d} c_{z}^{\prime}
\end{aligned}
$$

and

$$
\begin{aligned}
h\left(a,-c_{x}, c_{y}, c_{z}\right)= & \alpha_{2} \delta_{2}\left(c^{2}-2\right)+\left(1-\alpha_{2}\right) h\left(a, c_{x}, c_{y}, c_{z}\right) \\
& +\frac{2 \alpha_{2}}{\pi} \int_{-\infty}^{\infty} \int_{-\infty}^{\infty} \int_{0}^{\infty} \mathrm{e}^{-c^{\prime 2}} h\left(a, c_{x}^{\prime}, c_{y}^{\prime}, c_{z}^{\prime}\right) c_{x}^{\prime} \mathrm{d} c_{x}^{\prime} \mathrm{d} c_{y}^{\prime} \mathrm{d} c_{z}^{\prime}
\end{aligned}
$$

with,

$$
\delta_{i}=\frac{T_{\omega i}-T_{0}}{T_{0}}, \quad i=1,2
$$

for $T_{0}$ a reference temperature, which usually is assumed as $\left(T_{\omega 1}+T_{\omega 2}\right) / 2$. We note that, to guarantee physically meaningful values for the temperatures one must assumes $\delta_{i} \geq-1$, for $i=1,2$.

In writing the boundary conditions above, we consider that a fraction of the particles $\alpha$ is reflected diffusely and the rest $(1-\alpha)$ specularly. In each of the walls, $\alpha_{i} \in(0,1], i=1,2$, denotes the accommodation coefficient.

\subsection{The temperature-jump problem}

In this work, we consider also the problem of evaluating the temperature jump at the wall located at $\tau=0$. In this case, in addition to the boundary condition defined for $c_{x}>0$ as

$$
\begin{aligned}
h\left(0, c_{x}, c_{y}, c_{z}\right)=(1 & -\alpha) h\left(0,-c_{x}, c_{y}, c_{z}\right) \\
& +\frac{2 \alpha}{\pi} \int_{-\infty}^{\infty} \int_{-\infty}^{\infty} \int_{0}^{\infty} \mathrm{e}^{-c^{\prime 2}} h\left(0,-c_{x}^{\prime}, c_{y}^{\prime}, c_{z}^{\prime}\right) c_{x}^{\prime} \mathrm{d} c_{x}^{\prime} \mathrm{d} c_{y}^{\prime} \mathrm{d} c_{z}^{\prime},
\end{aligned}
$$

to solve Eq. (5), we impose the Welander condition $[12,28]$ on the perturbation of the temperature

$$
\lim _{\tau \rightarrow \infty} \frac{\mathrm{d}}{\mathrm{d} \tau} T(\tau)=\mathcal{K}
$$

where $\mathcal{K}$ is constant. We still note that, in Eq. (18), again, $\alpha \in(0,1]$ is the accommodation coefficient. 


\section{Basic formulation}

Since the quantities we want to evaluate, Eqs. (13) to (15), are defined in terms of moments of the $h$ function, we look for simpler problems written in terms of those moments. In this way, we first define

$$
g_{1}\left(\tau, c_{x}\right)=\pi^{-1} \int_{-\infty}^{\infty} \int_{-\infty}^{\infty} \mathrm{e}^{-\left(c_{y}^{2}+c_{z}^{2}\right)} h\left(\tau, c_{x}, c_{y}, c_{z}\right) \mathrm{d} c_{y} \mathrm{~d} c_{z}
$$

and

$$
g_{2}\left(\tau, c_{x}\right)=\pi^{-1} \int_{-\infty}^{\infty} \int_{-\infty}^{\infty}\left(c_{y}^{2}+c_{z}^{2}-1\right) \mathrm{e}^{-\left(c_{y}^{2}+c_{z}^{2}\right)} h\left(\tau, c_{x}, c_{y}, c_{z}\right) \mathrm{d} c_{y} \mathrm{~d} c_{z} .
$$

Then, we multiply Eq. (5) by

$$
\phi_{1}\left(c_{y}, c_{z}\right)=\pi^{-1} \mathrm{e}^{-\left(c_{y}^{2}+c_{z}^{2}\right)}
$$

and we integrate over all $c_{y}$ and $c_{z}$, such that, (introducing the new notation $\left.c_{x}=\xi\right)$ we obtain a new equation for $g_{1}$

$$
\begin{aligned}
\xi \frac{\partial}{\partial \tau} g_{1}(\tau, \xi) & +\varepsilon g_{1}(\tau, \xi) \\
& =\varepsilon \pi^{-1 / 2} \int_{-\infty}^{\infty} \mathrm{e}^{-\xi^{\prime 2}}\left[k_{11}\left(\xi^{\prime}, \xi\right) g_{1}\left(\tau, \xi^{\prime}\right)+k_{12}\left(\xi^{\prime}, \xi\right) g_{2}\left(\tau, \xi^{\prime}\right)\right] \mathrm{d} \xi^{\prime},
\end{aligned}
$$

where

$$
\begin{aligned}
& k_{11}\left(\xi^{\prime}, \xi\right)=1+2 \xi \xi^{\prime} \\
& \quad+(2 / 3)\left(\xi^{2}-1 / 2\right)\left(\xi^{\prime 2}-1 / 2\right)+(4 / 5) \beta \xi \xi^{\prime}\left(\xi^{2}-3 / 2\right)\left(\xi^{\prime 2}-3 / 2\right) \\
& +2 \varpi\left[(2 / 3) \xi^{2} \xi^{\prime 2}-(1 / 3)\left(\xi^{2}+\xi^{\prime 2}\right)+1 / 6\right]
\end{aligned}
$$

and

$$
k_{12}\left(\xi^{\prime}, \xi\right)=(2 / 3)\left(\xi^{2}-1 / 2\right)+(4 / 5) \beta \xi \xi^{\prime}\left(\xi^{2}-3 / 2\right)+2 \varpi\left[1 / 6-(1 / 3) \xi^{2}\right] .
$$

Following analogous procedure, we multiply Eq. (5) now by

$$
\phi_{2}\left(c_{y}, c_{z}\right)=\pi^{-1}\left(c_{y}^{2}+c_{z}^{2}-1\right) \mathrm{e}^{-\left(c_{y}^{2}+c_{z}^{2}\right)}
$$

and we integrate over all $c_{y}$ and $c_{z}$ to obtain (for $c_{x}=\xi$ )

$$
\begin{aligned}
\xi \frac{\partial}{\partial \tau} g_{2}(\tau, \xi) & +\varepsilon g_{2}(\tau, \xi) \\
& =\varepsilon \pi^{-1 / 2} \int_{-\infty}^{\infty} \mathrm{e}^{-\xi^{\prime 2}}\left[k_{21}\left(\xi^{\prime}, \xi\right) g_{1}\left(\tau, \xi^{\prime}\right)+k_{22}\left(\xi^{\prime}, \xi\right) g_{2}\left(\tau, \xi^{\prime}\right)\right] \mathrm{d} \xi^{\prime}
\end{aligned}
$$

with

$$
k_{21}\left(\xi^{\prime}, \xi\right)=(2 / 3)\left(\xi^{\prime 2}-1 / 2\right)+(4 / 5) \beta \xi \xi^{\prime}\left(\xi^{\prime 2}-3 / 2\right)+2 \varpi\left[1 / 6-(1 / 3) \xi^{\prime 2}\right]
$$


and

$$
k_{22}\left(\xi^{\prime}, \xi\right)=2 / 3+(4 / 5) \beta \xi \xi^{\prime}+(1 / 3) \varpi .
$$

Eqs. (23) and (27) can be rewritten in a vector form as

$$
\xi \frac{\partial}{\partial \tau} \mathbf{G}(\tau, \xi)+\varepsilon \mathbf{G}(\tau, \xi)=\varepsilon \int_{-\infty}^{\infty} \psi\left(\xi^{\prime}\right) \mathbf{K}\left(\xi^{\prime}, \xi\right) \mathbf{G}\left(\tau, \xi^{\prime}\right) \mathrm{d} \xi^{\prime},
$$

where $\mathbf{G}(\tau, \xi)$ has components $g_{1}(\tau, \xi)$ and $g_{2}(\tau, \xi)$,

$$
\psi(\xi)=\pi^{-1 / 2} \mathrm{e}^{-\xi^{2}}
$$

and the components $k_{i j}\left(\xi^{\prime}, \xi\right)$ of the $2 \times 2$ matrix $\mathbf{K}\left(\xi^{\prime}, \xi\right)$ are defined in Eqs. $(24)$, (25), (28) and (29).

Analogously, the boundary conditions given by Eqs. (16) are rewritten, for $\xi>0$, as

$$
\mathbf{G}(-a, \xi)=\left(1-\alpha_{1}\right) \mathbf{G}(-a,-\xi)+2 \alpha_{1} \boldsymbol{\Gamma} \int_{0}^{\infty} \mathbf{G}\left(-a,-\xi^{\prime}\right) \mathrm{e}^{-\xi^{\prime 2}} \xi^{\prime} \mathrm{d} \xi^{\prime}+\alpha_{1} \delta_{1} \boldsymbol{\Omega}(\xi)
$$

and

$$
\mathbf{G}(a,-\xi)=\left(1-\alpha_{2}\right) \mathbf{G}(a, \xi)+2 \alpha_{2} \boldsymbol{\Gamma} \int_{0}^{\infty} \mathbf{G}\left(a, \xi^{\prime}\right) \mathrm{e}^{-\xi^{\prime 2}} \xi^{\prime} \mathrm{d} \xi^{\prime}+\alpha_{2} \delta_{2} \boldsymbol{\Omega}(\xi)
$$

with

$$
\boldsymbol{\Gamma}=\left[\begin{array}{ll}
1 & 0 \\
0 & 0
\end{array}\right]
$$

and

$$
\boldsymbol{\Omega}(\xi)=\left[\begin{array}{c}
\xi^{2}-1 \\
1
\end{array}\right]
$$

In the temperature-jump problem, the boundary condition given by Eq. (18) is written, in the vector form, by

$$
\mathbf{G}(0, \xi)-(1-\alpha) \mathbf{G}(0,-\xi)-2 \alpha \boldsymbol{\Gamma} \int_{0}^{\infty} \mathbf{G}\left(0,-\xi^{\prime}\right) \mathrm{e}^{-\xi^{\prime 2}} \xi^{\prime} \mathrm{d} \xi^{\prime}=\mathbf{0} .
$$

In terms of the definitions given in Eqs. (20) and (21) the quantities of interest are also expressed in a vector form. In this way, the solution for the $\mathbf{G}(\tau, \xi)$ problem is used to evaluate the density perturbation distribution

$$
N(\tau)=\int_{-\infty}^{\infty} \psi(\xi)\left[\begin{array}{l}
1 \\
0
\end{array}\right]^{T} \mathbf{G}(\tau, \xi) \mathrm{d} \xi
$$

the temperature perturbation

$$
T(\tau)=\frac{2}{3} \int_{-\infty}^{\infty} \psi(\xi)\left[\begin{array}{c}
\xi^{2}-1 / 2 \\
1
\end{array}\right]^{T} \mathbf{G}(\tau, \xi) \mathrm{d} \xi
$$


and the heat flow

$$
Q_{x}=\int_{-\infty}^{\infty} \psi(\xi) \xi\left[\begin{array}{c}
\xi^{2}-3 / 2 \\
1
\end{array}\right]^{T} \mathbf{G}(\tau, \xi) \mathrm{d} \xi
$$

At this point, we also define, for the heat-transfer problem, a normalized heat flow $[10]$ as

$$
q=\frac{Q_{x}}{Q_{f m}}
$$

where $Q_{f m}$ is the dimensionless heat flow for free-molecules

$$
Q_{f m}=\frac{\alpha_{1} \alpha_{2}\left(\delta_{1}-\delta_{2}\right)}{\left(\alpha_{1}+\alpha_{2}-\alpha_{1} \alpha_{2}\right) \pi^{1 / 2}} .
$$

Looking back to Eqs. (38) to (40), we write the normalized heat flow in terms of the solution of the problem given by Eq. (30), and the boundary conditions given by Eqs. (32), as

$$
q=\frac{1}{Q_{f m}} \int_{-\infty}^{\infty} \psi(\xi) \xi\left[\begin{array}{c}
\xi^{2}-3 / 2 \\
1
\end{array}\right]^{T} \mathbf{G}(\tau, \xi) \mathrm{d} \xi
$$

We develop the solution for the $\mathbf{G}$ problem in the next section.

\section{A discrete ordinates solution}

Firstly, looking back to Eq. (31) and noting that $\psi(\xi)$ is an even function, we rewrite the integral term in Eq. (30),

$$
\xi \frac{\partial}{\partial \tau} \mathbf{G}(\tau, \xi)+\varepsilon \mathbf{G}(\tau, \xi)=\varepsilon \int_{0}^{\infty} \psi\left(\xi^{\prime}\right)\left[\mathbf{K}\left(\xi^{\prime}, \xi\right) \mathbf{G}\left(\tau, \xi^{\prime}\right)+\mathbf{K}\left(-\xi^{\prime}, \xi\right) \mathbf{G}\left(\tau,-\xi^{\prime}\right)\right] \mathrm{d} \xi^{\prime} .
$$

Then we introduce a (half-range) quadrature scheme in $[0, \infty)$, to approximate the integral term of the above equation, such that

$$
\xi \frac{\partial}{\partial \tau} \mathbf{G}(\tau, \xi)+\varepsilon \mathbf{G}(\tau, \xi)=\varepsilon \sum_{k=1}^{N} \omega_{k} \psi\left(\xi_{k}\right)\left[\mathbf{K}\left(\xi_{k}, \xi\right) \mathbf{G}\left(\tau, \xi_{k}\right)+\mathbf{K}\left(-\xi_{k}, \xi\right) \mathbf{G}\left(\tau,-\xi_{k}\right)\right] .
$$

Here, $\left\{\xi_{k}, \omega_{k}\right\}$ are, respectively, the $N$ nodes and weights of the (arbitrary) quadrature scheme. If we now evaluate Eq. (43) in $\xi= \pm \xi_{i}$, for $i=1, \ldots, N$, we obtain the discrete-ordinates version of Eq. (42) as

$$
\begin{aligned}
\pm \xi_{i} \frac{\mathrm{d}}{\mathrm{d} \tau} \mathbf{G}\left(\tau, \pm \xi_{i}\right)+\varepsilon \mathbf{G}\left(\tau, \pm \xi_{i}\right) \\
\quad=\varepsilon \sum_{k=1}^{N} \omega_{k} \psi\left(\xi_{k}\right)\left[\mathbf{K}\left(\xi_{k}, \pm \xi_{i}\right) \mathbf{G}\left(\tau, \xi_{k}\right)+\mathbf{K}\left(-\xi_{k}, \pm \xi_{i}\right) \mathbf{G}\left(\tau,-\xi_{k}\right)\right]
\end{aligned}
$$


to which we seek solutions of the form

$$
\mathbf{G}(\tau, \xi)=\boldsymbol{\Phi}(\nu, \xi) \mathrm{e}^{-\varepsilon \tau / \nu},
$$

where

$$
\boldsymbol{\Phi}(\nu, \xi)=\left[\begin{array}{l}
\Phi_{1}(\nu, \xi) \\
\Phi_{2}(\nu, \xi)
\end{array}\right]
$$

If we substitute Eq. (45) into Eq. (44) we obtain, for $i=1, \ldots, N$,

$$
\left(1 \mp \xi_{i} / \nu\right) \boldsymbol{\Phi}\left(\nu, \pm \xi_{i}\right)=\sum_{k=1}^{N} \omega_{k} \psi\left(\xi_{k}\right)\left[\mathbf{K}\left(\xi_{k}, \pm \xi_{i}\right) \boldsymbol{\Phi}\left(\nu, \xi_{k}\right)+\mathbf{K}\left(-\xi_{k}, \pm \xi_{i}\right) \boldsymbol{\Phi}\left(\nu,-\xi_{k}\right)\right],
$$

and we can write Eqs. (47) in a matrix form as

$$
\left(\mathbf{I}-\nu^{-1} \mathbf{M}\right) \boldsymbol{\Phi}_{+}(\nu)=\mathbf{W}(+,+) \boldsymbol{\Phi}_{+}(\nu)+\mathbf{W}(-,+) \boldsymbol{\Phi}_{-}(\nu)
$$

and

$$
\left(\mathbf{I}+\nu^{-1} \mathbf{M}\right) \boldsymbol{\Phi}_{-}(\nu)=\mathbf{W}(+,-) \boldsymbol{\Phi}_{+}(\nu)+\mathbf{W}(-,-) \boldsymbol{\Phi}_{-}(\nu),
$$

where $\mathbf{I}$ is the $2 N \times 2 N$ identity matrix, $\mathbf{M}$ is the $2 N \times 2 N$ matrix defined as

$$
\mathbf{M}=\operatorname{diag}\left\{\xi_{1}, \xi_{2}, \ldots, \xi_{N}, \xi_{1}, \xi_{2}, \ldots, \xi_{N}\right\},
$$

and $\boldsymbol{\Phi}_{ \pm}(\nu)$ are $2 N \times 1$ vectors, such that

$$
\boldsymbol{\Phi}_{ \pm}(\nu)=\left[\begin{array}{llllll}
\Phi_{1}\left(\nu, \pm \xi_{1}\right) & \cdots & \Phi_{1}\left(\nu, \pm \xi_{N}\right) & \Phi_{2}\left(\nu, \pm \xi_{1}\right) & \cdots & \Phi_{2}\left(\nu, \pm \xi_{N}\right)
\end{array}\right]^{T} .
$$

Continuing, $T$ denotes transpose operation, and $\mathbf{W}( \pm, \pm)$ are $2 N \times 2 N$ matrices, with $N \times N$ submatrices components

$$
\mathbf{W}( \pm, \pm)=\left[\begin{array}{ll}
\mathbf{W}_{11}( \pm, \pm) & \mathbf{W}_{\mathbf{1 2}}( \pm, \pm) \\
\mathbf{W}_{\mathbf{2 1}}( \pm, \pm) & \mathbf{W}_{\mathbf{2 2}}( \pm, \pm)
\end{array}\right]
$$

where the $N \times N$ submatrices are defined, after noting Eqs. (24), (25), (28), (29) and (31), as

$$
\left[\mathbf{W}_{m n}( \pm, \pm)\right]_{i, j}=\omega_{j} \psi\left(\xi_{j}\right) k_{m n}\left( \pm \xi_{j}, \pm \xi_{i}\right)
$$

for $m, n=1,2$ and $i, j=1, \ldots, N$.

From Eqs. (24), (25), (28) and (29) we find that, the kernel in Eq. (30) is such that

$$
\mathbf{K}\left(\xi^{\prime}, \xi\right)=\mathbf{K}\left(-\xi^{\prime},-\xi\right)
$$

and

$$
\mathbf{K}\left(-\xi^{\prime}, \xi\right)=\mathbf{K}\left(\xi^{\prime},-\xi\right) .
$$

In addition, from Eqs. (24), (25), (28), (29), it is also possible to see, in Eq. (51) that

$$
\mathbf{W}(+,+)=\mathbf{W}(-,-)
$$

and

$$
\mathbf{W}(+,-)=\mathbf{W}(-,+) .
$$


In this way, from now on, we define the matrices

$$
\mathbf{W}_{+}=\mathbf{W}(+,+)=\mathbf{W}(-,-)
$$

and

$$
\mathbf{W}_{-}=\mathbf{W}(+,-)=\mathbf{W}(-,+)
$$

and we rewrite Eqs. (48) as

$$
\left(\mathbf{I}-\nu^{-1} \mathbf{M}\right) \boldsymbol{\Phi}_{+}(\nu)=\mathbf{W}_{+} \boldsymbol{\Phi}_{+}(\nu)+\mathbf{W}_{-} \boldsymbol{\Phi}_{-}(\nu)
$$

and

$$
\left(\mathbf{I}+\nu^{-1} \mathbf{M}\right) \boldsymbol{\Phi}_{-}(\nu)=\mathbf{W}_{-} \boldsymbol{\Phi}_{+}(\nu)+\mathbf{W}_{+} \boldsymbol{\Phi}_{-}(\nu) .
$$

Now, we first let

$$
\mathbf{U}=\boldsymbol{\Phi}_{+}(\nu)+\boldsymbol{\Phi}_{-}(\nu),
$$

where $\boldsymbol{\Phi}_{+}(\nu)$ and $\boldsymbol{\Phi}_{-}(\nu)$ are $2 N \times 1$ vectors defined in Eq. (50), and we then add and subtract Eqs. (56) such that we are able to define an eigenvalue problem

$$
\mathbf{A X}=\lambda \mathbf{X}
$$

Here, $\mathbf{A}$ is the $2 N \times 2 N$ matrix

$$
\mathbf{A}=\left(\mathbf{W}_{+}-\mathbf{W}_{-}-\mathbf{I}\right) \mathbf{M}^{-1}\left(\mathbf{W}_{+}+\mathbf{W}_{-}-\mathbf{I}\right) \mathbf{M}^{-\mathbf{1}}
$$

and, noting Eq. (49), $\mathbf{X}$ is the $2 N \times 1$ vector

$$
\mathbf{X}=\mathbf{M U} \text {. }
$$

Here, the (real) eigenvalues are related to the separation constants as

$$
\lambda=\nu^{-2} \text {. }
$$

In fact, we use the $2 N$ eigenvalues (separation constants $\nu_{j}$ ) and the $2 N$ eigenvectors $\mathbf{X}\left(\nu_{j}\right)$ given by Eq. (58) to express the elementary solutions written in Eqs. (45) and (50) in the form

$$
\boldsymbol{\Phi}_{+}\left(\nu_{j}\right)=\frac{1}{2} \mathbf{M}^{-\mathbf{1}}\left[\mathbf{I}-\nu_{j}\left(\mathbf{W}_{+}+\mathbf{W}_{-}-\mathbf{I}\right) \mathbf{M}^{-\mathbf{1}}\right] \mathbf{X}\left(\nu_{j}\right)
$$

and

$$
\Phi_{-}\left(\nu_{j}\right)=\frac{1}{2} \mathbf{M}^{-\mathbf{1}}\left[\mathbf{I}+\nu_{j}\left(\mathbf{W}_{+}+\mathbf{W}_{-}-\mathbf{I}\right) \mathbf{M}^{-\mathbf{1}}\right] \mathbf{X}\left(\nu_{j}\right)
$$

In this way, we are ready to write the general solution of the discrete-ordinates problem given by Eq. (44) as

$$
\mathbf{G}\left(\tau, \pm \xi_{i}\right)=\sum_{j=1}^{2 N}\left[A_{j} \boldsymbol{\Phi}\left(\nu_{j}, \pm \xi_{i}\right) \mathrm{e}^{-\varepsilon(a+\tau) / \nu_{j}}+B_{j} \boldsymbol{\Phi}\left(\nu_{j}, \mp \xi_{i}\right) \mathrm{e}^{-\varepsilon(a-\tau) / \nu_{j}}\right],
$$

where the vectors $\boldsymbol{\Phi}\left(\nu_{j}, \pm \xi_{i}\right)$, are given in Eq. (46).

At this point it is important to emphasize that, except for the defining expressions of the components of the kernel - Eqs. (24), (25), (28) and (29) - which are 
entries of the matrix written in Eqs. (51) and (52), the eigenvalue problem we find here, in Eq. (58) is exactly the same one we obtained when the ADO solution was developed for flow problems [41]. The defining expressions given in Eqs. (62) and (63), are also the same. We see this is a good aspect of the ADO solution, when one thinks of implementing the solution for obtaining numerical results.

Now, since these are conservative problems, we have to deal with the issue of having eigenvalues that approach zero (separation constants going to infinity) as $N$ tends to infinity. For these two specific problems we are solving in this work, we find two eigenvalues with that behavior. Because of that, we disregard the associated elementary solutions and we add four exact solutions of the problem given by Eq. (30), to the general solution given in Eq. (64), which we rewrite in the form

$$
\begin{aligned}
\mathbf{G}\left(\tau, \pm \xi_{i}\right)= & \mathbf{G}^{*}\left(\tau, \pm \xi_{i}\right) \\
& +\sum_{j=1}^{2 N-2}\left[A_{j} \boldsymbol{\Phi}\left(\nu_{j}, \pm \xi_{i}\right) \mathrm{e}^{-\varepsilon(a+\tau) / \nu_{j}}+B_{j} \boldsymbol{\Phi}\left(\nu_{j}, \mp \xi_{i}\right) \mathrm{e}^{-\varepsilon(a-\tau) / \nu_{j}}\right]
\end{aligned}
$$

where,

$$
\mathbf{G}^{*}(\tau, \xi)=A_{1}^{*} \mathbf{G}_{1}+A_{2}^{*} \mathbf{G}_{2}(\xi)+B_{1}^{*} \mathbf{G}_{3}(\xi)+B_{2}^{*} \mathbf{G}_{4}(\tau, \xi),
$$

and, for the temperature-jump problem we set $a=0$ in Eq. (65). Here, the introduced exact solutions are

$$
\mathbf{G}_{1}=\left[\begin{array}{l}
1 \\
0
\end{array}\right], \quad \mathbf{G}_{2}(\xi)=\left[\begin{array}{c}
\xi^{2}-1 / 2 \\
1
\end{array}\right], \quad \mathbf{G}_{3}(\xi)=\left[\begin{array}{l}
\xi \\
0
\end{array}\right]
$$

and

$$
\mathbf{G}_{4}(\tau, \xi)=\tau \mathbf{H}(\xi)+\mathbf{F}(\xi)
$$

with

$$
\mathbf{H}(\xi)=\left[\begin{array}{c}
\xi^{2}-3 / 2 \\
1
\end{array}\right] \text { and } \mathbf{F}(\xi)=\frac{\xi}{\varepsilon(\beta-1)}\left[\begin{array}{c}
\xi^{2}-3 / 2 \\
1
\end{array}\right] .
$$

The next step is to determine the arbitrary constants present in the solution, Eq. (65), which will be done in the next section, by using the boundary conditions. Before doing that, however, we want to emphasize two aspects. Firstly, regarding to the eigenvalue problem derived here, expressed in Eq. (58), which includes the $\mathrm{S}$ model case: the derivation here presented, for the ADO method, is simpler than the one described for the temperature-jump problem, for the S model in Ref. [30], which related the one gas and the binary gas mixture cases.

Secondly, a special remark has to be done with respect to derivation of the exact solutions given in Eqs. (67), which are, for instance, different of the flow problems case [41]. In earlier works where the ADO method was used for solving (scalar) problems in this field of rarefied gas dynamics $[49,37]$ the derivation of the needed exact solutions was based on the procedure already proposed by Case and Zweifel [50] for conservative problems in neutron transport. The procedure 
had to be extended for the components of vector problems [8, 27, 28]. In fact, the derivation of the exact solutions is an issue to be investigated depending on the problem. Here, the exact solutions introduced are variations of previous works $[8,27,51,30]$.

In particular, to derive the solution given in Eq. (67d), we follow a procedure proposed by Siewert [51] where, once we know $\mathbf{H}(\xi)$, we substitute Eq. (67d) into Eq. (30) to obtain

$$
\mathbf{F}(\xi)=-(\xi / \varepsilon) \mathbf{H}(\xi)+\int_{-\infty}^{\infty} \psi\left(\xi^{\prime}\right) \mathbf{K}\left(\xi^{\prime}, \xi\right) \mathbf{F}\left(\xi^{\prime}\right) \mathrm{d} \xi^{\prime} .
$$

Following Refs. [51, 30], we write

$$
\mathbf{F}(\xi)=\sum_{\alpha=0}^{3} P_{\alpha}(\xi) \mathbf{F}_{\alpha}
$$

where

$$
P_{0}(\xi)=1, \quad P_{1}(\xi)=\xi, \quad P_{2}(\xi)=\xi^{2}-1 / 2 \quad \text { and } \quad P_{3}(\xi)=\xi\left(\xi^{2}-3 / 2\right) .
$$

We then substitute Eq. (69) into Eq. (68), we multiply the resulting equation by $\psi(\xi) P_{k}(\xi)$, for $k=0,1,2,3$, and we integrate over all $\xi$, to obtain a $8 \times 8$ linear system which, in this case, can be solved analytically (differently of the mixture case) to find the components of the vectors $\mathbf{F}_{\alpha}$.

Having explained the two points above, as we mention before our next step is to obtain the $4 N$ arbitrary constants $A_{1}^{*}, A_{2}^{*}, B_{1}^{*}, B_{2}^{*}$ and $A_{j}, B_{j}, j=1, \ldots, 2 N-2$ for each one of the two problems we are interested in this work, in order to define physical quantities of interest.

\section{Physical quantities of interest}

\subsection{The heat-transfer problem}

We consider the discrete-ordinates version of the boundary conditions given in Eqs. (32)

$$
\mathbf{G}\left(-a, \xi_{i}\right)=\left(1-\alpha_{1}\right) \mathbf{G}\left(-a,-\xi_{i}\right)+2 \alpha_{1} \boldsymbol{\Gamma} \sum_{k=1}^{N} \omega_{k} \xi_{k} \mathbf{G}\left(-a,-\xi_{k}\right) \mathrm{e}^{-\xi_{k}^{2}}+\alpha_{1} \delta_{1} \boldsymbol{\Omega}\left(\xi_{i}\right)
$$

and

$$
\mathbf{G}\left(a,-\xi_{i}\right)=\left(1-\alpha_{2}\right) \mathbf{G}\left(a, \xi_{i}\right)+2 \alpha_{2} \boldsymbol{\Gamma} \sum_{k=1}^{N} \omega_{k} \xi_{k} \mathbf{G}\left(a, \xi_{k}\right) \mathrm{e}^{-\xi_{k}^{2}}+\alpha_{2} \delta_{2} \boldsymbol{\Omega}\left(\xi_{i}\right),
$$

where we will apply the general solution, Eq. (65). However, we note that, since the solution $\mathbf{G}_{1}$, Eq. (67a), satisfies the homogeneous boundary condition, the 
coefficient $A_{1}^{*}$ can not be determined. We then solve, using the least-squares approach, a $4 N$ equations and $4 N-1$ unknowns linear system defined (noting Eqs. (67)) by the equations

$$
\begin{aligned}
& \alpha_{1} A_{2}^{*} \boldsymbol{\Omega}\left(\xi_{i}\right)+\sum_{j=1}^{2 N-2} A_{j}\left\{\boldsymbol{\Phi}\left(\nu_{j}, \xi_{i}\right)-\left(1-\alpha_{1}\right) \boldsymbol{\Phi}\left(\nu_{j},-\xi_{i}\right)-2 \alpha_{1} \mathbf{G}_{1} \mathbf{S}_{-}\left(\nu_{j}\right)\right\} \\
& \quad+B_{1}^{*}\left[\left(2-\alpha_{1}\right) \mathbf{G}_{3}\left(\xi_{i}\right)+\left(\alpha_{1} / 2\right) \pi^{1 / 2} \mathbf{G}_{1}\right]+B_{2}^{*}\left[\left(2-\alpha_{1}\right) \mathbf{F}\left(\xi_{i}\right)-\alpha_{1} a \boldsymbol{\Omega}\left(\xi_{i}\right)\right] \\
& +\sum_{j=1}^{2 N-2} B_{j}\left\{\boldsymbol{\Phi}\left(\nu_{j},-\xi_{i}\right)-\left(1-\alpha_{1}\right) \boldsymbol{\Phi}\left(\nu_{j}, \xi_{i}\right)-2 \alpha_{1} \mathbf{G}_{1} \mathbf{S}_{+}\left(\nu_{j}\right)\right\} \mathrm{e}^{-2 a \varepsilon / \nu_{j}}=\alpha_{1} \delta_{1} \boldsymbol{\Omega}\left(\xi_{i}\right)
\end{aligned}
$$

and

$$
\begin{gathered}
\alpha_{2} A_{2}^{*} \boldsymbol{\Omega}\left(\xi_{i}\right)+\sum_{j=1}^{2 N-2} A_{j}\left\{\boldsymbol{\Phi}\left(\nu_{j},-\xi_{i}\right)-\left(1-\alpha_{2}\right) \boldsymbol{\Phi}\left(\nu_{j}, \xi_{i}\right)-2 \alpha_{2} \mathbf{G}_{1} \mathbf{S}_{+}\left(\nu_{j}\right)\right\} \mathrm{e}^{-2 a \varepsilon / \nu_{j}} \\
\quad-B_{1}^{*}\left[\left(2-\alpha_{2}\right) \mathbf{G}_{3}\left(\xi_{i}\right)+\left(\alpha_{2} / 2\right) \pi^{1 / 2} \mathbf{G}_{1}\right]-B_{2}^{*}\left[\left(2-\alpha_{2}\right) \mathbf{F}\left(\xi_{i}\right)-\alpha_{2} a \boldsymbol{\Omega}\left(\xi_{i}\right)\right] \\
+\sum_{j=1}^{2 N-2} B_{j}\left\{\boldsymbol{\Phi}\left(\nu_{j}, \xi_{i}\right)-\left(1-\alpha_{2}\right) \boldsymbol{\Phi}\left(\nu_{j},-\xi_{i}\right)-2 \alpha_{2} \mathbf{G}_{1} \mathbf{S}_{-}\left(\nu_{j}\right)\right\}=\alpha_{2} \delta_{2} \boldsymbol{\Omega}\left(\xi_{i}\right), \quad(72 \mathrm{~b})
\end{gathered}
$$

for $i=1, \ldots, N$. Here $\boldsymbol{\Phi}\left(\nu_{j}, \pm \xi_{i}\right)$ are defined in Eq. (46),

$$
\mathbf{S}_{-}\left(\nu_{j}\right)=\sum_{k=1}^{N} \omega_{k} \xi_{k} \mathrm{e}^{-\xi_{k}^{2}}\left[\begin{array}{l}
1 \\
0
\end{array}\right]^{T} \boldsymbol{\Phi}\left(\nu_{j},-\xi_{k}\right)
$$

and

$$
\mathbf{S}_{+}\left(\nu_{j}\right)=\sum_{k=1}^{N} \omega_{k} \xi_{k} \mathrm{e}^{-\xi_{k}^{2}}\left[\begin{array}{l}
1 \\
0
\end{array}\right]^{T} \boldsymbol{\Phi}\left(\nu_{j}, \xi_{k}\right) .
$$

The discrete-ordinates solution of Eq. (30), with boundary conditions given by Eqs. (32), is now completely established, except by the coefficient $A_{1}^{*}$. We are able to write, however, the physical quantities of interest for the heat-transfer problem. In this way, we apply Eq. (65) into Eqs. (36) and (37) to obtain, respectively, the density perturbation and the temperature perturbation

$$
N(\tau)=A_{1}^{*}-B_{2}^{*} \tau+\sum_{j=1}^{2 N-2}\left[A_{j} \mathrm{e}^{-\varepsilon(a+\tau) / \nu_{j}}+B_{j} \mathrm{e}^{-\varepsilon(a-\tau) / \nu_{j}}\right] \mathbf{V}\left(\nu_{j}\right)
$$

and

$$
T(\tau)=A_{2}^{*}+B_{2}^{*} \tau+\frac{2}{3} \sum_{j=1}^{2 N-2}\left[A_{j} \mathrm{e}^{-\varepsilon(a+\tau) / \nu_{j}}+B_{j} \mathrm{e}^{-\varepsilon(a-\tau) / \nu_{j}}\right] \mathbf{Y}\left(\nu_{j}\right)
$$


Here

$$
\mathbf{V}\left(\nu_{j}\right)=\sum_{k=1}^{N} \omega_{k} \psi\left(\xi_{k}\right)\left[\begin{array}{l}
1 \\
0
\end{array}\right]^{T}\left[\mathbf{\Phi}\left(\nu_{j}, \xi_{k}\right)+\mathbf{\Phi}\left(\nu_{j},-\xi_{k}\right)\right]
$$

and

$$
\mathbf{Y}\left(\nu_{j}\right)=\sum_{k=1}^{N} \omega_{k} \psi\left(\xi_{k}\right)\left[\begin{array}{c}
\xi_{k}^{2}-1 / 2 \\
1
\end{array}\right]^{T}\left[\mathbf{\Phi}\left(\nu_{j}, \xi_{k}\right)+\boldsymbol{\Phi}\left(\nu_{j},-\xi_{k}\right)\right] .
$$

We note that, the density perturbation depends on the coefficient $A_{1}^{*}$, and so, it is not uniquely defined. Because of that, we follow Ref. [8] and use the normalization condition

$$
\int_{-a}^{a} N(\tau) \mathrm{d} \tau=0
$$

such that, if we substitute Eq. (74) into Eq. (78) we get

$$
A_{1}^{*}=\frac{1}{2 a \varepsilon} \sum_{j=1}^{2 N-2} \nu_{j}\left(\mathrm{e}^{-2 a \varepsilon / \nu_{j}}-1\right)\left(A_{j}+B_{j}\right) \mathbf{V}\left(\nu_{j}\right)
$$

Now, looking back to Eq. (38) we can also define the heat flow

$$
Q_{x}=\frac{5}{4 \varepsilon(\beta-1)} B_{2}^{*}+\sum_{j=1}^{2 N-2}\left[A_{j} \mathrm{e}^{-\varepsilon(a+\tau) / \nu_{j}}-B_{j} \mathrm{e}^{-\varepsilon(a-\tau) / \nu_{j}}\right] \mathbf{Z}\left(\nu_{j}\right),
$$

where

$$
\mathbf{Z}\left(\nu_{j}\right)=\sum_{k=1}^{N} \omega_{k} \xi_{k} \psi\left(\xi_{k}\right)\left[\begin{array}{c}
\xi_{k}^{2}-3 / 2 \\
1
\end{array}\right]^{T}\left[\mathbf{\Phi}\left(\nu_{j}, \xi_{k}\right)-\mathbf{\Phi}\left(\nu_{j},-\xi_{k}\right)\right] .
$$

It is noted, however, that the summation in Eq. (80) goes to zero as $N$ increases, and so, it is usual to restrict the evaluation of the normalized heat flow, expressed in Eq. (41), to

$$
q=\frac{5}{4 \varepsilon(\beta-1) Q_{f m}} B_{2}^{*},
$$

although it was not analytically proved. Here $Q_{f m}$ is given by Eq. (40).

\subsection{The temperature-jump problem}

For this half-space problem, in addition to the discrete-ordinates version of the boundary conditions given by Eq. (35)

$$
\mathbf{G}\left(0, \xi_{i}\right)-(1-\alpha) \mathbf{G}\left(0,-\xi_{i}\right)-2 \alpha \boldsymbol{\Gamma} \sum_{k=1}^{N} \omega_{k} \xi_{k} \mathbf{G}\left(0,-\xi_{k}\right) \mathrm{e}^{-\xi_{k}^{2}}=\mathbf{0},
$$


we consider the Welander condition as written in Eq. (19). In this way, we impose the solution does not increase exponentially at infinity, such that, for $j=1, \ldots, 2 N-2$ we take $B_{j}=0$. In other words, we write the general solution, Eq. (65), for $a=0$, as

$$
\mathbf{G}\left(\tau, \pm \xi_{i}\right)=\mathbf{G}^{*}\left(\tau, \pm \xi_{i}\right)+\sum_{j=1}^{2 N-2} A_{j} \boldsymbol{\Phi}\left(\nu_{j}, \pm \xi_{i}\right) \mathrm{e}^{-\varepsilon \tau / \nu_{j}}
$$

Continuing, we see that, if we apply Eq. (84) into Eq. (37) and use Eq. (19) we get

$$
B_{2}^{*}=\mathcal{K}
$$

We then substitute Eq. (84) into Eq. (83) to obtain the $2 N \times 2 N$ linear system for the other arbitrary constants in the following form

$$
\begin{aligned}
\sum_{j=1}^{2 N-2} A_{j}\left\{\boldsymbol{\Phi}\left(\nu_{j}, \xi_{i}\right)\right. & \left.-(1-\alpha) \boldsymbol{\Phi}\left(\nu_{j},-\xi_{i}\right)-2 \alpha \mathbf{G}_{1} \mathbf{S}\left(\nu_{j}\right)\right\}+\alpha A_{2}^{*} \boldsymbol{\Omega}\left(\xi_{i}\right) \\
& +B_{1}^{*}\left[(2-\alpha) \mathbf{G}_{3}\left(\xi_{i}\right)+(\alpha / 2) \pi^{1 / 2} \mathbf{G}_{1}\right]=(\alpha-2) \mathcal{K} \mathbf{F}\left(\xi_{i}\right)
\end{aligned}
$$

for $i=1, \ldots, N$. Here again, $\boldsymbol{\Phi}\left(\nu_{j}, \pm \xi_{i}\right)$ are defined in Eq. (46) and

$$
\mathbf{S}\left(\nu_{j}\right)=\sum_{k=1}^{N} \omega_{k} \xi_{k} \mathrm{e}^{-\xi_{k}^{2}}\left[\begin{array}{l}
1 \\
0
\end{array}\right]^{T} \boldsymbol{\Phi}\left(\nu_{j},-\xi_{k}\right) .
$$

Once more, since $\mathbf{G}_{1}$ satisfies the boundary condition, Eq. (35), we are not able to evaluate the coefficient $A_{1}^{*}$. Now, if we use Eq. (84) into Eqs. (36) and (37), and note Eqs. (76) and (77), we see that the density perturbation

$$
N(\tau)=A_{1}^{*}-\mathcal{K} \tau+\sum_{j=1}^{2 N-2} A_{j} \mathrm{e}^{-\varepsilon \tau / \nu_{j}} \mathbf{V}\left(\nu_{j}\right)
$$

depends on that coefficient, and, on the other hand, it does not affect the temperature perturbation

$$
T(\tau)=A_{2}^{*}+\mathcal{K} \tau+\frac{2}{3} \sum_{j=1}^{2 N-2} A_{j} \mathrm{e}^{-\varepsilon \tau / \nu_{j}} \mathbf{Y}\left(\nu_{j}\right) .
$$

Following previous work [28] we impose the normalization condition

$$
\lim _{\tau \rightarrow \infty}[N(\tau)+T(\tau)]=0
$$

to find that $A_{1}^{*}=-A_{2}^{*}$ and to rewrite the density perturbation in the final form

$$
N(\tau)=-A_{2}^{*}-\mathcal{K} \tau+\sum_{j=1}^{2 N-2} A_{j} \mathrm{e}^{-\varepsilon \tau / \nu_{j}} \mathbf{V}\left(\nu_{j}\right) .
$$


Now, if we consider the linear component in Eq. (89)

$$
T_{a s y}(\tau)=A_{2}^{*}+\mathcal{K} \tau,
$$

we can use it to define the temperature-jump coefficient $\zeta[12]$, which relates the temperature perturbation at the wall with the normal gradient of the temperature, as

$$
T_{a s y}(0)=\left.\zeta \frac{\mathrm{d}}{\mathrm{d} \tau} T_{a s y}(\tau)\right|_{\tau=0}
$$

such that

$$
\zeta=A_{2}^{*} / \mathcal{K}
$$

\section{Computational procedures and numerical results}

To implement the ADO solution, the first step is to define the quadrature scheme. As we have explained in previous works, in general, we define a half-range quadrature scheme in $[0, \infty)$. We use the transformation

$$
u(\xi)=\mathrm{e}^{-\xi}
$$

to map the interval $[0, \infty)$ into $[0,1]$, where we are then able to use the Gauss Legendre usual scheme with a new change of variable:

$$
v(u)=2 u-1 .
$$

The numerical results showed here, were obtained by two independent FORTRAN programs where, once we have the $N$ quadrature points $\xi_{k}$ and the weights $\omega_{k}$ defined, the main steps to follow are simple:

- to solve an eigenvalue problem, Eq. (58), to obtain the separation constants $\nu_{j}$ and the elementary solutions $\boldsymbol{\Phi}_{ \pm}\left(\nu_{j}\right)$

- to solve a linear system, given by Eqs. (72) for the heat-transfer problem and Eq. (86) for the temperature-jump problem.

- to evaluate the temperature and density perturbations by Eqs. (74) and (75) - or Eqs. (89) and (91) - as well as, the normalized heat flow (Eq. (82)) and the jump coefficient (Eq. (94)).

In this sense, the solution is concise, easy to implement and fast (less than one second in a $2.66 \mathrm{GHz}$ Pentium IV machine). We obtain the results presented in this work using $N=50$ quadrature points, since we noted that all the digits listed here are preserved (plus or minus one in the last digit) when we increase the value of $N$ (up to 100).

Some parameters are needed to be defined in order to get the numerical results. In particular, we use the constant $\mathcal{K}=1$, in Eq. (19). In the case of $\varepsilon$, as mentioned previously, different values are assumed, according the specific kinetic model [45] and, depending on the definition of the mean-free-path in terms of viscosity $\left(\varepsilon_{p}\right)$ or thermal conductivity $\left(\varepsilon_{t}\right)$. These values are listed in Eqs. (8) to (11). 
To have some confidence in our programs and also in order to be able to perform a general comparative analysis, initially we defined and solved some cases to which results were available in the literature. In fact, we note that results for the BGK $[8,27]$ and S model [30] previously known, and used to check our results, can be obtained in our formulation for an appropriate choice of the parameters in Eq. (7a). We found good agreement with results listed (with one less digit than we list in our tables) in those works. In this way, for the heat-transfer problem, we present in Tables 1 to 6 , a complete comparison between results obtained in this work for all the models, and the linearized Boltzmann equation (LBE) [10]. In Ref. [10] it was used $\varepsilon=\varepsilon_{t}$. Results for density perturbation, temperature perturbation and heat flow are showed, for different values of channel width, accommodation coefficients and temperatures imposed at the wall. We note agreement, between the kinetic models and the LBE, in 1 to 3 digits, in general. Many simulations were performed and a more complete and detailed analysis is presented in Refs. [26] and [52]. Differently of the case of flow problems [41], it seems difficult to say, for this problem, which kinetic model produces better results, when compared with LBE results. But it seems that more agreement is obtained mostly by the $\mathrm{S}$ model formulation.

For the temperature-jump problem, we first check our program comparing results for the BGK and S model with results available in previous works [27, 30]. In this case, in Tables 20 to 22, the results obtained in this work, were compared with the CES model [29] and the LBE equation [35]. Same general aspects noted in regard to the previous problem are observed here, and again we emphasize that in Refs. [29] and [35], it was used $\varepsilon=\varepsilon_{t}$.

In addition to that, we generate for both problems some new results, in order to establish a better analysis in regard to the possible influence of certain parameters (as the accommodation coefficients, the temperature at the walls, the channel thickness) on the quantities of interest. In Tables 7 to 19 these results are showed for the heat-transfer problem. In Tables 23 to 26, for the temperature-jump problem.

Along with the results presented in the tables, we generate some figures, in order to help the analysis (under a different point of view) of the influence of some parameters in the behavior of the quantities of interest. In this way, temperature perturbation and heat flow, as well as, the temperature-jump coefficient are analyzed in Figs. 1 to 6 . In particular, $\varepsilon=\varepsilon_{t}$ was used to generate the $\mathrm{S}$ model results used to generate the figures.

For the heat-transfer problem, we can note that (see Tables 11 to 14) variation in the accommodation coefficients seems to produce more significant effects on the temperature distribution than the variation on the imposed temperature at the walls, as possible expected surface effects in micro scale. It can be noted, from the analysis of the slopes of the curves, in Figs. 1 and 2. In fact, we can see in Fig. 4, nonlinear dependence on accommodation coefficients for the heat flow.

For the temperature-jump problem we can also note, from the showed results, 


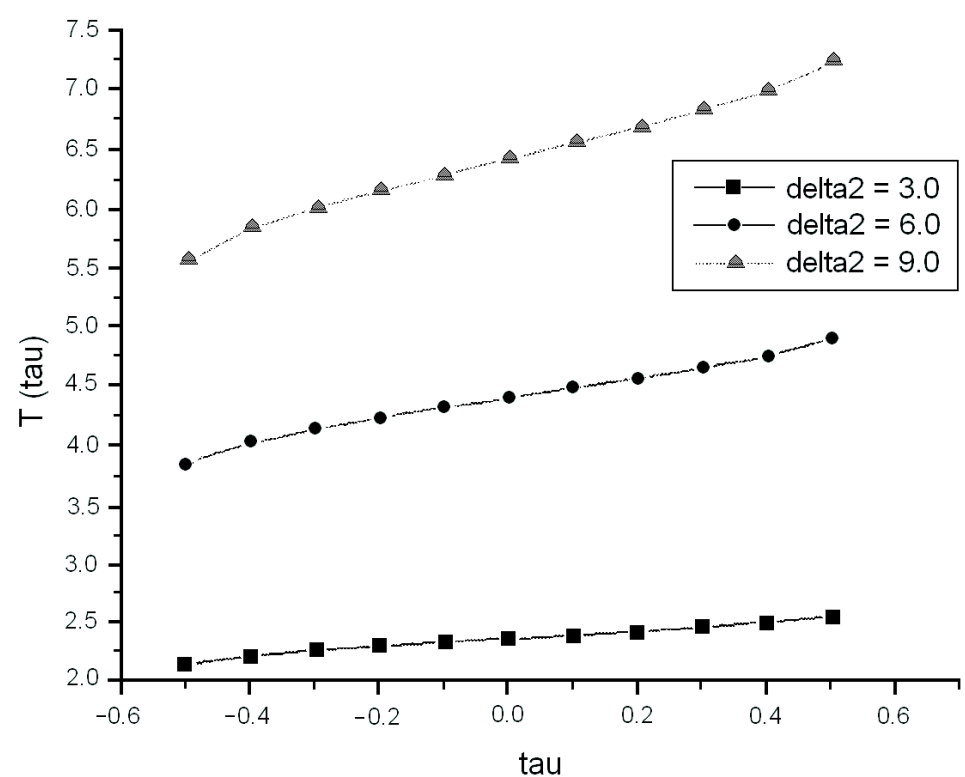

Figure 1. Heat transfer problem: temperature perturbation $T(\tau), 2 a=1.0, \alpha_{1}=0.3, \alpha_{2}=0.6$, $\delta_{1}=1.0$

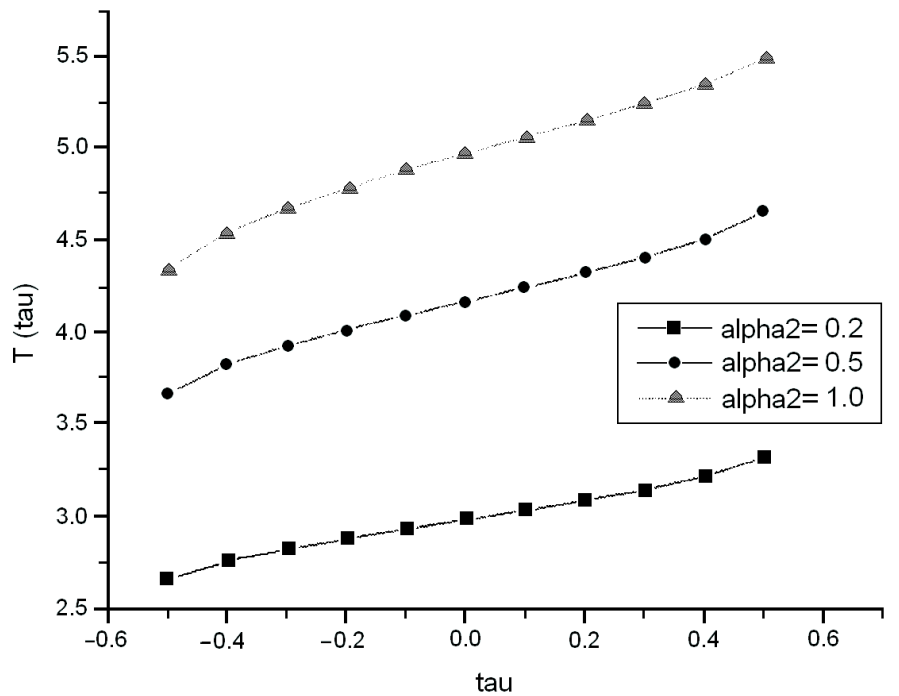

Figure 2. Heat transfer problem: temperature perturbation $T(\tau), 2 a=1.0, \delta_{1}=1.0, \delta_{2}=6.0$, $\alpha_{1}=0.3$ 


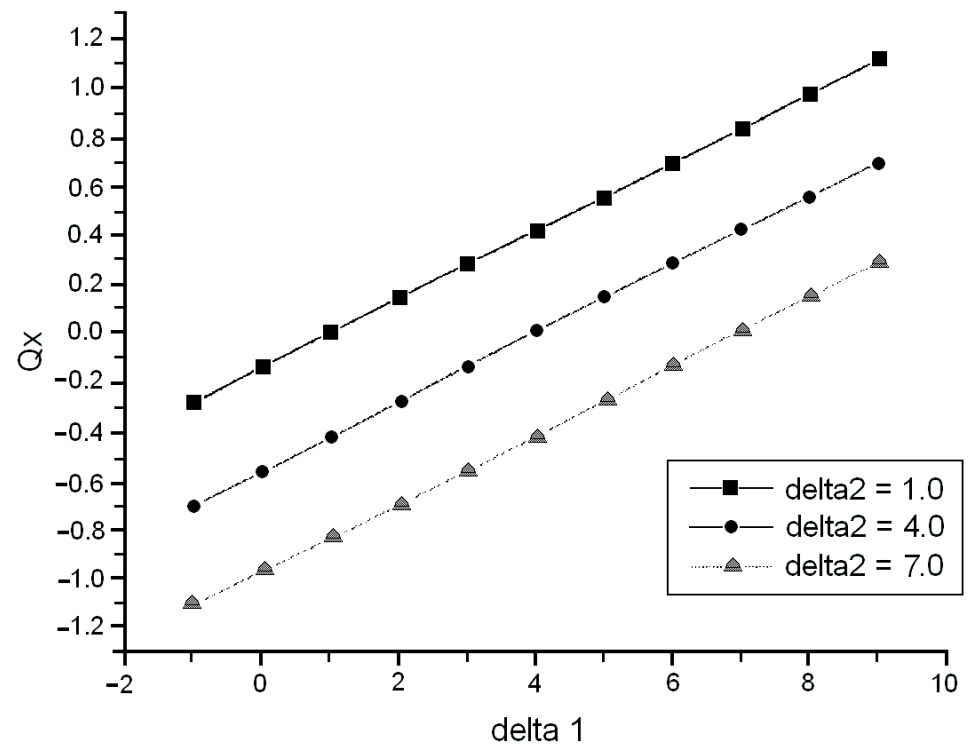

Figure 3. Heat transfer problem: heat flow $Q_{x}, 2 a=1.0, \alpha_{1}=0.9, \alpha_{2}=0.3$

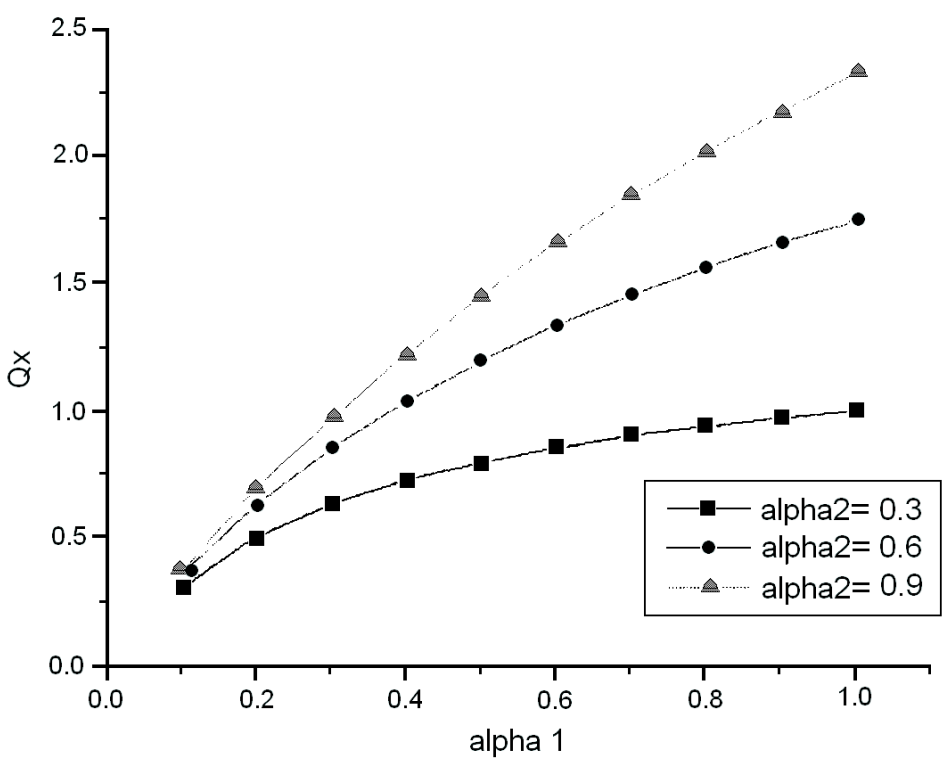

Figure 4. Heat transfer problem: heat flow $Q_{x}, 2 a=1.0, \delta_{1}=8.0, \delta_{2}=1.0$ 


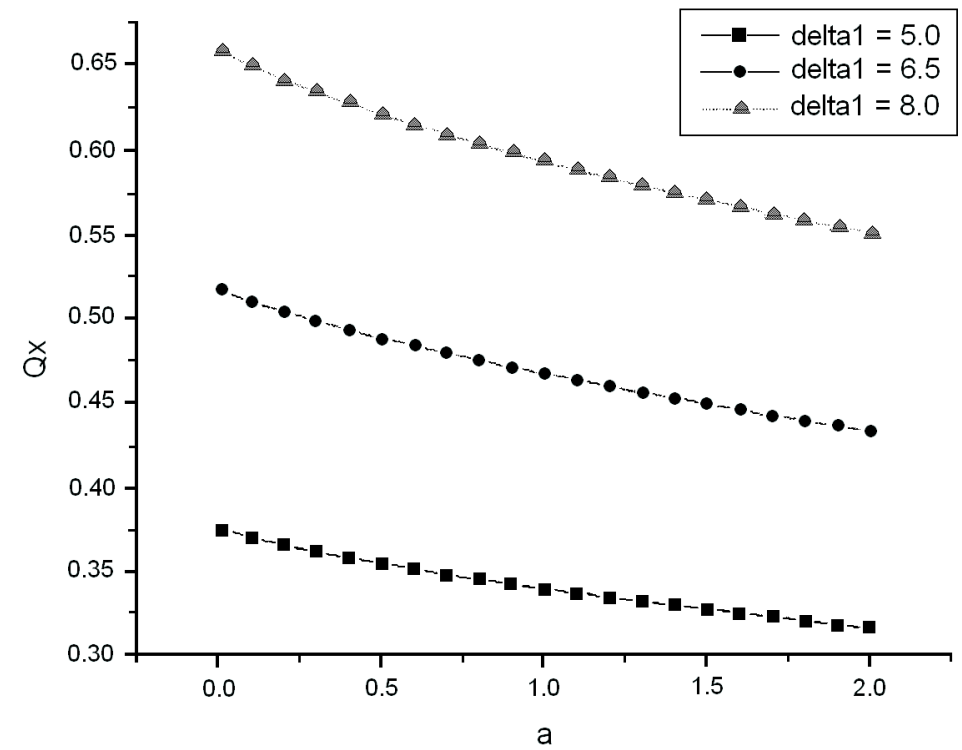

Figure 5. Heat transfer problem: heat flow $Q_{x}, \alpha_{1}=0.2, \alpha_{2}=0.5, \delta_{2}=1.0$

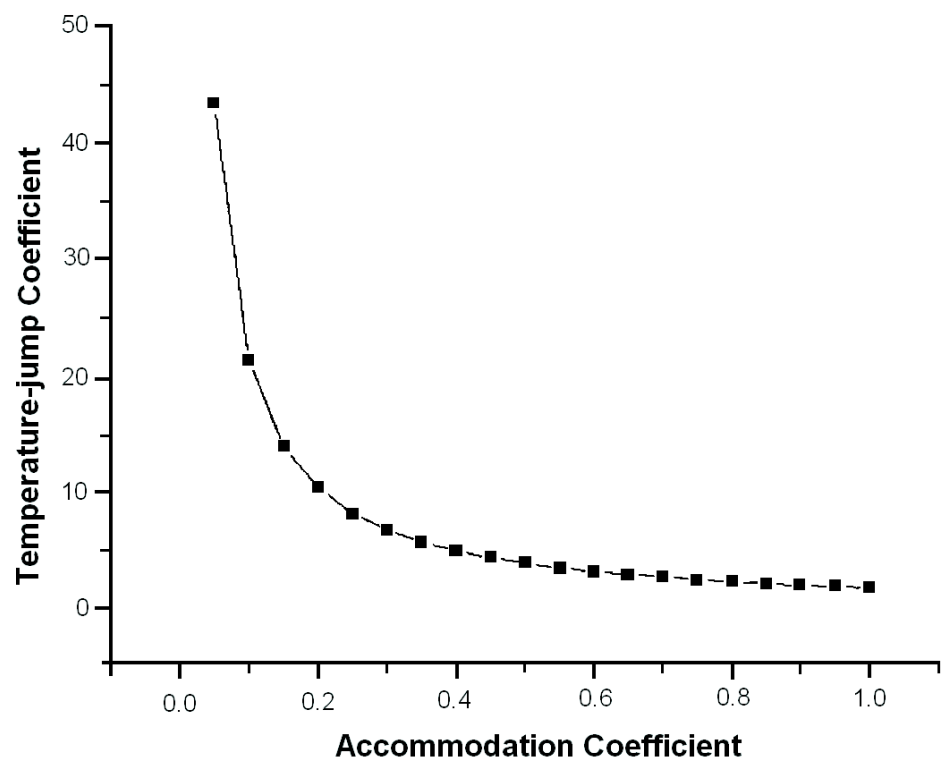

Figure 6. Temperature jump problem: the temperature-jump coefficient $\zeta$ 
the effect of the accommodation coefficient on the order of magnitude for the jump, which increases significantly (see Fig. 6) in the range far from the perfect accommodation.

\section{Concluding comments}

In this work, the solution of the heat-transfer problem for a rarefied gas confined in a plane channel was analyzed, as well as the evaluation of the temperature jump at the wall. An analytical discrete-ordinates solution was developed, by the ADO method, for a class of four kinetic equations in a general procedure. The results showed a good agreement between the kinetic model results and results available for the LBE equation. In addition, through the accommodation coefficients, it was analyzed the special influence of the surface effects on the physical quantities of interest. In subsequent paper, we show that, evaporation effects can be also taking into account following the same basic procedure, which emphasizes the general aspect of this analytical approach.

\section{Acknowledgement}

This work is partially supported by CNPq - Conselho Nacional de Desenvolvimento Científico e Tecnológico - of Brazil.

\section{References}

[1] E. P. Gross and S. Ziering, Heat Flow Between Parallel Plates, The Phys. of Fluids 2 (1959), 701.

[2] K. Frankowski, Z. Alterman and C. L. Pekeris, Heat Transport Between Parallel Plates in a Rarefied Gas of Rigid Sphere Molecules, The Phys. of Fluids 8 (1965), 245.

[3] P. Bassanini, C. Cercignani and C. D. Pagani, Comparison of Kinetic Theory Analysis of Linearized Heat Transfer Between Parallel Plates, Int. J. of Heat and Mass Transfer 10 (1966), 447.

[4] P. Bassanini, C. Cercignani and C. D. Pagani, Influence of the Accommodation Coefficient on the Heat Transfer in a Rarefied Gas, Int. J. of Heat and Mass Transfer 11 (1968), 1359.

[5] J. R. Thomas Jr., T. S. Chang and C. E. Siewert, Heat Transfer Between Parallel Plates with Arbitrary Surface Accommodation, The Phys. of Fluids 16 (1973), 2116.

[6] D. Valougeorgis and J. R. Thomas Jr., The $F_{N}$ Method in Kinetic Theory: II. Heat Transfer between Parallel Plates, Transp. Theory and Stat. Phys. 14 (1985), 497.

[7] T. Ohwada, K. Aoki and Y. Sone, Heat Transfer and Temperature Distribution in a Rarefied Gas Between Two Parallel Plates with Different Temperatures: Numerical Analysis of the Boltzmann Equation for a Hard Sphere Molecule, Rarefied Gas Dynamics: Theoretical and Computational Techniques, edited by E. P. Muntz, D. P. Weaver and D. H. Campbell, Vol. 118 of Progress in Astronautics and Aeronautics, AIAA, Washington, DC (1989).

[8] C. E. Siewert, A Discrete-Ordinates Solution for Heat Transfer in a Plane Channel, J. of Comp. Phys. 152 (1999), 251. 
[9] K. Aoki, S. Takata, H. Aikawa and F. Golse, A Rarefied Gas Flow Caused by a Discontinuous Wall Temperature, Phys. Fluids 13 (2001), 2645.

[10] C. E. Siewert, Heat Transfer and Evaporation/Condensation Problems Based on the Linearized Boltzmann Equation, Euro. J. Mech. B/Fluids 22 (2003), 391.

[11] R. D. M. Garcia and C. E. Siewert, The McCormack Model for Gas Mixtures: Heat Transfer in a Plane Channel, Phys. Fluids 16 (2004), 3393.

[12] P. Welander, On the Temperature Jump in a Rarefied Gas, Arkiv Fysik 7 (1954), 507.

[13] Y. Onishi, Effects of Accommodation Coefficient on Temperature and Density Fields in a Slightly Rarefied Gas, Transactions Japan Soc. Aero. Space Sciences 17 (1974), 151.

[14] S. K. Loyalka, Temperature Jump and Thermal Creep Slip: Rigid Sphere Gas, Phys. Fluids A 1 (1989), 403.

[15] Y. Sone, T. Ohwada and K. Aoki, Temperature Jump and Knudsen Layer in Rarefied Gas Over a Plane Wall: Numerical Analysis of the Linearized Boltzmann Equation for HardSphere Molecules, Phys. Fluids A 1 (1989), 363.

[16] C. Cercignani and M. Lampis, Variational Calculation of the Temperature Jump for a Binary Mixture, Rarefied Gas Dynamics, edited by A. E. Beylich, VCH, 1991. p. 1379.

[17] F. Sharipov, Application of the Cercignani-Lampis Scattering Kernel to Calculations of Rarefied Gas Flows. II. Slip and Jump Coefficients, Euro. J. Mech. B/Fluids 22 (2003), 133.

[18] M. Gad-El-Hak, The MEMS Handbook, CRC Press, Boca Raton 2006.

[19] H. Struchtrup, Heat Transfer in the Transition Regime: Solution of Boundary Value Problems for Grad's Moment Equations Via Kinetic Schemes, Phys. Rev. E 65 (2002), 041204.

[20] R. Raju and S. Roy, Hydrodynamic Study of High-Speed Flow and Heat Transfer Through a Microchannel, J. Thermophysics and Heat Transfer 19 (2005), 106.

[21] G. Karniadakis, A. Beskok and N. Aluru, Microflows and Nanoflows, Springer, New York 2005.

[22] M. Le and I. Hassan, Simulation of Heat Transfer in High Speed Microflows, App. Thermal Eng. 26 (2006), 2035.

[23] M. M. R. Williams, Mathematical Methods in Particle Transport Theory, Butterworth, London 1971.

[24] C. Cercignani, The Boltzmann Equation and its Applications, Springer-Verlag, New York 1988.

[25] Y. Sone, Kinetic Theory and Fluid Dynamics, Birkhäuser, Boston 2002.

[26] C. S. Scherer, Kinetic Models of the Linearized Boltzmann Equation and a Heat Transfer Problem in Microscale (In Portuguese), MSc. Dissertation, Programa de Pós-Graduação em Matemática Aplicada, Universidade Federal do Rio Grande do Sul, Porto Alegre, RS, Brasil 2005.

[27] L. B. Barichello and C. E. Siewert, The Temperature-Jump Problem in Rarefied Gas Dynamics, Euro. J. Applied Math. 11 (2000), 353.

[28] L. B. Barichello, A. C. R. Bartz, M. Camargo and C. E. Siewert, The Temperature-Jump Problem for a Variable Collision Frequency Model, Phys. Fluids 14 (2002), 382.

[29] C. E. Siewert, The Temperature-Jump Problem Based on the CES Model of the Linearized Boltzmann Equation, Z. Angew. Math. Phys. 55 (2004), 92.

[30] R. F. Knackfuss and L. B. Barichello, On the Temperature-Jump Problem in Rarefied Gas Dynamics: the Effect of the Cercignani-Lampis Boundary Condition, SIAM J. Appl. Math. 66 (2006), 2149.

[31] L. B. Barichello and C. E. Siewert, A Discrete-Ordinates Solution for a Non-grey Model with Complete Frequency Redistribution, JQSRT 62 (1999), 665.

[32] S. Chandrasekhar, Radiative Transfer, Dover, New York 1960.

[33] P. L. Bhatnagar, E. P. Gross and M. Krook, A Model for Collision Processes in Gases.I. Small Amplitude Processes in Charged and Neutral One-Component Systems, Phys. Rev. 94 (1954), 511. 
[34] E. M. Shakhov, Generalization of the Krook Kinetic Relaxation Equation, Fluid Dynamics 3 (1968), 142. (In Russian)

[35] C. E. Siewert, The Linearized Boltzmann Equation: a Concise and Accurate Solution of the Temperature-jump Problem, JQSRT 77 (2003), 417.

[36] R. D. M. Garcia and C. E. Siewert, The Temperature-jump Problem Based on the Linearized Boltzmann Equation for a Binary Mixture of Rigid Spheres, Euro. J. of Mech. B/Fluids 26 (2007), 132.

[37] L. B. Barichello, M. Camargo, P. Rodrigues and C. E. Siewert, Unified Solutions to Classical Flow Problems Based on the BGK Model, Z. Angew. Math. Phys. 52 (2001), 1.

[38] C. E. Siewert, Poiseuille, Thermal Creep and Couette Flow: Results Based on the CES Model of the Linearized Boltzmann Equation, Euro. J. Mech. B/Fluids 21 (2002), 579.

[39] M. Camargo and L. B. Barichello, Unified Approach for Variable Collision Frequency Models in Rarefied Gas Dynamics, Transp. Theory and Stat. Phys. 33 (2004), 227.

[40] L. C. Cabrera and L. B. Barichello, Unified Solutions to Some Classical Problems in Rarefied Gas Dynamics Based on the One-dimensional Linearized S-model Equations, Z. Angew. Math. Phys. 57 (2006), 285.

[41] C. S. Scherer, J. F. Prolo Filho and L. B. Barichello, An Analytical Approach to the Unified Solution of Kinetic Equations in the Rarefied Gas Dynamics. I. Flow Problems, Z. Angew. Math. Phys., in press.

[42] E. P. Gross and E. A. Jackson, Kinetic Models and the Linearized Boltzmann Equation, The Phys. of Fluids 2 (1959), 432.

[43] R. D. M. Garcia and C. E. Siewert, The Linearized Boltzmann Equation: Sound-wave Propagation in a Rarefied Gas, Z. Angew. Math. Phys. 57 (2006), 94.

[44] M. M. R. Williams, A Review of the Rarefied Gas Dynamics Theory Associated With Some Classical Problems in Flow and Heat Transfer, Z. Angew. Math. Phys. 52 (2001), 500.

[45] L. B. Barichello and C. E. Siewert, Some Comments on Modeling the Linearized Boltzmann Equation, JQSRT 77 (2003), 43.

[46] C. E. Siewert, Generalized Boundary Conditions for the S-Model Kinetic Equations Basic to Flow in a Plane Channel, JQSRT 72 (2002), 75.

[47] C. E. Siewert and F. Sharipov, Model Equations in Rarefied Gas Dynamics: Viscous-Slip and Thermal-Slip Coefficients, Phys. Fluids 14 (2002), 4123.

[48] C. L. Pekeris and Z. Alterman, Solution of the Boltzmann-Hilbert Integral Equation II. The Coefficients of Viscosity and Heat Conduction, Proc. Natl. Acad. Science 43 (1957), 998.

[49] L. B. Barichello and C. E. Siewert, A Discrete-Ordinates Solution for Poiseuille Flow in a Plane Channel, Z. Angew. Math. Phys. 50 (1999), 972.

[50] K. M. Case and P. F. Zweifel, Linear Transport Theory, Addison-Wesley Publishing Company, Reading 1967.

[51] C. E. Siewert, The McCormack Model for Gas Mixtures: The Temperature-jump Problem, Z. Angew. Math. Phys. 56 (2005), 273.

[52] J. F. Prolo Filho, The Boltzmann Equation Modeling Flows in Micro and Macro Scales (In Portuguese), MSc. Dissertation, Programa de Pós-Graduação em Matemática Aplicada, Universidade Federal do Rio Grande do Sul, Porto Alegre, RS, Brasil 2007. 

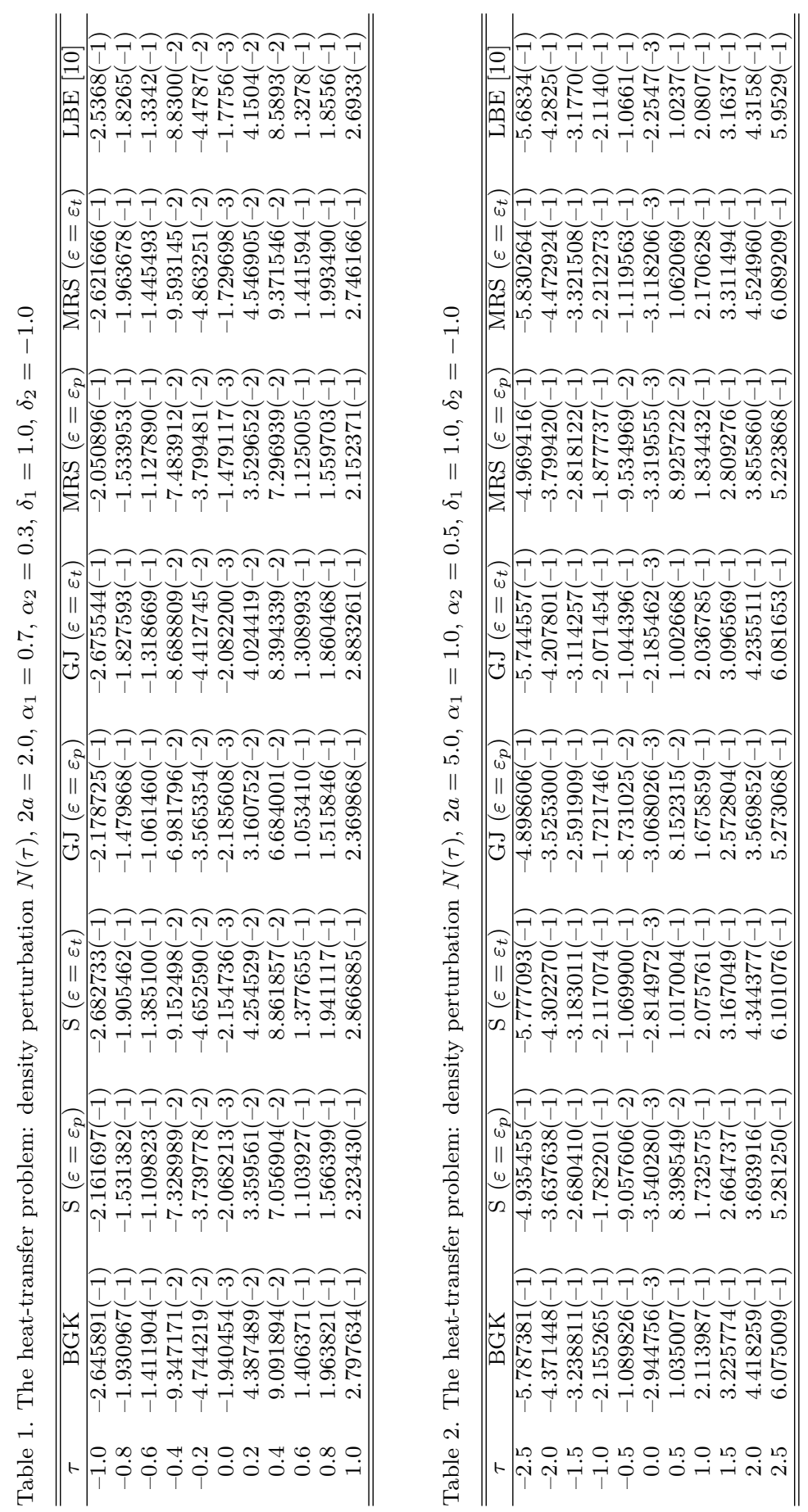

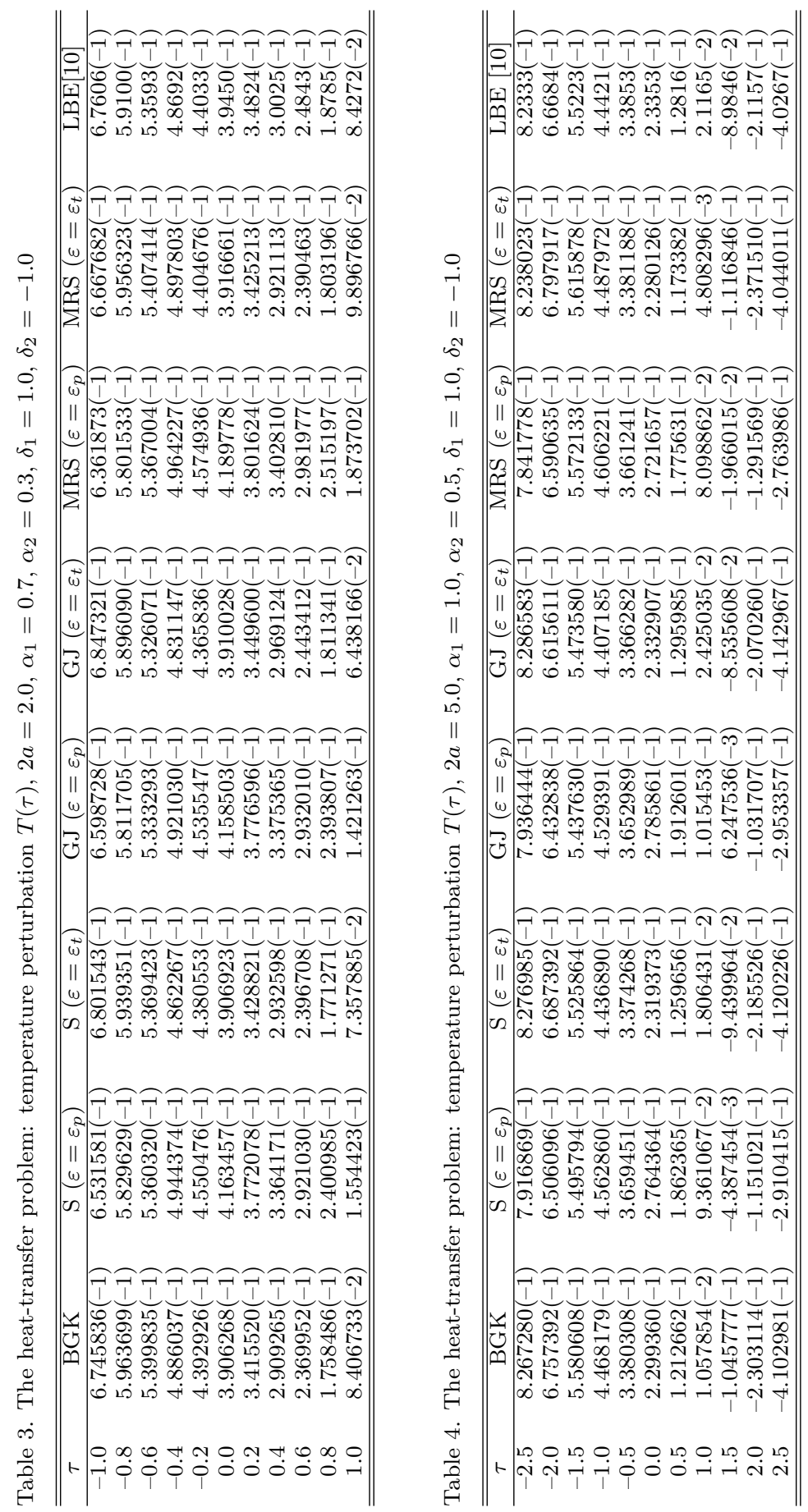
Table 5. The heat-transfer problem: normalized heat flow $q, \varepsilon=\varepsilon_{t}, 2 a=5.0, \delta_{1}=1.0$,

\begin{tabular}{ccccccc}
\multicolumn{7}{c}{$\delta_{2}=-1.0$} \\
\hline \hline$\alpha_{1}$ & $\alpha_{2}$ & BGK & S & GJ & MRS & LBE [10] \\
\hline 0.7 & 0.1 & $8.042633(-1)$ & $8.035306(-1)$ & $8.030294(-1)$ & $8.058217(-1)$ & $8.08046(-1)$ \\
0.7 & 0.3 & $5.990574(-1)$ & $5.980539(-1)$ & $5.973904(-1)$ & $6.011983(-1)$ & $6.04641(-1)$ \\
0.7 & 0.5 & $4.929175(-1)$ & $4.919910(-1)$ & $4.913948(-1)$ & $4.949030(-1)$ & $4.98494(-1)$ \\
0.7 & 0.7 & $4.282278(-1)$ & $4.274347(-1)$ & $4.269340(-1)$ & $4.299409(-1)$ & $4.33435(-1)$ \\
0.7 & 0.9 & $3.847899(-1)$ & $3.841333(-1)$ & $3.837222(-1)$ & $3.862285(-1)$ & $3.89560(-1)$ \\
0.7 & 1.0 & $3.680461(-1)$ & $3.674549(-1)$ & $3.670844(-1)$ & $3.693550(-1)$ & $3.72597(-1)$ \\
0.9 & 0.1 & $8.004407(-1)$ & $7.997988(-1)$ & $7.993510(-1)$ & $8.018463(-1)$ & $8.04152(-1)$ \\
0.9 & 0.3 & $5.794079(-1)$ & $5.785310(-1)$ & $5.779447(-1)$ & $5.813255(-1)$ & $5.84853(-1)$ \\
0.9 & 0.5 & $4.597632(-1)$ & $4.589723(-1)$ & $4.584625(-1)$ & $4.614917(-1)$ & $4.65054(-1)$ \\
0.9 & 0.7 & $3.847899(-1)$ & $3.841333(-1)$ & $3.837222(-1)$ & $3.862285(-1)$ & $3.89560(-1)$ \\
0.9 & 0.9 & $3.334255(-1)$ & $3.328995(-1)$ & $3.325767(-1)$ & $3.345867(-1)$ & $3.37630(-1)$ \\
0.9 & 1.0 & $3.133714(-1)$ & $3.129056(-1)$ & $3.126213(-1)$ & $3.144064(-1)$ & $3.17305(-1)$ \\
1.0 & 1.0 & $2.919178(-1)$ & $2.915091(-1)$ & $2.912618(-1)$ & $2.928302(-1)$ & $2.95558(-1)$ \\
\hline \hline
\end{tabular}

Table 6. The heat-transfer problem: normalized heat flow $q, \varepsilon=\varepsilon_{t}, 2 a=0.2, \delta_{1}=1.0$,

\begin{tabular}{ccccccc}
\multicolumn{7}{c}{$\delta_{2}=-1.0$} \\
\hline \hline$\alpha_{1}$ & $\alpha_{2}$ & BGK & S & GJ & MRS & LBE $[10]$ \\
\hline 0.7 & 0.1 & $9.866428(-1)$ & $9.848277(-1)$ & $9.825229(-1)$ & $9.880406(-1)$ & $9.85339(-1)$ \\
0.7 & 0.3 & $9.642502(-1)$ & $9.597169(-1)$ & $9.540464(-1)$ & $9.677859(-1)$ & $9.61123(-1)$ \\
0.7 & 0.5 & $9.462739(-1)$ & $9.398866(-1)$ & $9.320062(-1)$ & $9.513156(-1)$ & $9.42048(-1)$ \\
0.7 & 0.7 & $9.315774(-1)$ & $9.239249(-1)$ & $9.146055(-1)$ & $9.376882(-1)$ & $9.26730(-1)$ \\
0.7 & 0.9 & $9.193798(-1)$ & $9.108756(-1)$ & $9.006500(-1)$ & $9.262484(-1)$ & $9.14234(-1)$ \\
0.7 & 1.0 & $9.140383(-1)$ & $9.052258(-1)$ & $8.946962(-1)$ & $9.211968(-1)$ & $9.08832(-1)$ \\
0.9 & 0.1 & $9.862970(-1)$ & $9.844793(-1)$ & $9.821893(-1)$ & $9.877064(-1)$ & $9.85019(-1)$ \\
0.9 & 0.3 & $9.613244(-1)$ & $9.565784(-1)$ & $9.506960(-1)$ & $9.650564(-1)$ & $9.58139(-1)$ \\
0.9 & 0.5 & $9.391618(-1)$ & $9.322438(-1)$ & $9.238008(-1)$ & $9.446757(-1)$ & $9.34745(-1)$ \\
0.9 & 0.7 & $9.193798(-1)$ & $9.108756(-1)$ & $9.006500(-1)$ & $9.262484(-1)$ & $9.14234(-1)$ \\
0.9 & 0.9 & $9.016318(-1)$ & $8.920002(-1)$ & $8.805875(-1)$ & $9.095146(-1)$ & $8.96135(-1)$ \\
0.9 & 1.0 & $8.934300(-1)$ & $8.833759(-1)$ & $8.715497(-1)$ & $9.017137(-1)$ & $8.87870(-1)$ \\
1.0 & 1.0 & $8.836675(-1)$ & $8.731437(-1)$ & $8.608625(-1)$ & $8.924007(-1)$ & $8.78053(-1)$ \\
\hline \hline
\end{tabular}


Table 7. The heat-transfer problem: density perturbation $N(\tau)$, Gross-Jackson model, $\varepsilon=\varepsilon_{t}$, $2 a=1.0, \alpha_{1}=0.9, \alpha_{2}=0.1$

\begin{tabular}{rccccc}
\hline \hline & $\delta_{1}=8.0$ & $\delta_{1}=8.0$ & $\delta_{1}=8.0$ & $\delta_{1}=8.0$ & $\delta_{1}=8.0$ \\
$\tau$ & $\delta_{2}=1.0$ & $\delta_{2}=3.0$ & $\delta_{2}=5.0$ & $\delta_{2}=7.0$ & $\delta_{2}=9.0$ \\
\hline-0.5 & $-2.608418(-1)$ & $-1.863155(-1)$ & $-1.117893(-1)$ & $-3.726311(-2)$ & $3.726311(-2)$ \\
-0.4 & $-1.829596(-1)$ & $-1.306854(-1)$ & $-7.841127(-2)$ & $-2.613709(-2)$ & $2.613709(-2)$ \\
-0.3 & $-1.332247(-1)$ & $-9.516050(-2)$ & $-5.709630(-2)$ & $-1.903210(-2)$ & $1.903210(-2)$ \\
-0.2 & $-8.930586(-2)$ & $-6.378990(-2)$ & $-3.827394(-2)$ & $-1.275798(-2)$ & $1.275798(-2)$ \\
-0.1 & $-4.754407(-2)$ & $-3.396005(-2)$ & $-2.037603(-2)$ & $-6.792010(-3)$ & $6.792010(-3)$ \\
0.0 & $-6.179842(-3)$ & $-4.414172(-3)$ & $-2.648503(-3)$ & $-8.828345(-4)$ & $8.828345(-4)$ \\
0.1 & $3.615202(-2)$ & $2.582287(-2)$ & $1.549372(-2)$ & $5.164575(-3)$ & $-5.164575(-3)$ \\
0.2 & $8.106389(-2)$ & $5.790278(-2)$ & $3.474167(-2)$ & $1.158055(-2)$ & $-1.158055(-2)$ \\
0.3 & $1.312717(-1)$ & $9.376550(-2)$ & $5.625930(-2)$ & $1.875310(-2)$ & $-1.875310(-2)$ \\
0.4 & $1.933337(-1)$ & $1.380955(-1)$ & $8.285734(-2)$ & $2.761911(-2)$ & $-2.761911(-2)$ \\
0.5 & $3.110808(-1)$ & $2.222005(-1)$ & $1.333203(-1)$ & $4.444011(-2)$ & $-4.444011(-2)$ \\
\hline \hline
\end{tabular}

Table 8. The heat-transfer problem: density perturbation $N(\tau)$, MRS model, $\varepsilon=\varepsilon_{t}, 2 a=1.0$, $\alpha_{1}=0.9, \alpha_{2}=0.1$

\begin{tabular}{cccccc}
\hline \hline & $\delta_{1}=8.0$ & $\delta_{1}=8.0$ & $\delta_{1}=8.0$ & $\delta_{1}=8.0$ & \multicolumn{1}{c}{$\delta_{1}=8.0$} \\
$\tau$ & $\delta_{2}=1.0$ & $\delta_{2}=3.0$ & $\delta_{2}=5.0$ & $\delta_{2}=7.0$ & $\delta_{2}=9.0$ \\
\hline-0.5 & $-2.391373(-1)$ & $-1.708124(-1)$ & $-1.024874(-1)$ & $-3.416248(-2)$ & $3.416248(-2)$ \\
-0.4 & $-1.815600(-1)$ & $-1.296857(-1)$ & $-7.781145(-2)$ & $-2.593715(-2)$ & $2.593715(-2)$ \\
-0.3 & $-1.346948(-1)$ & $-9.621061(-2)$ & $-5.772637(-2)$ & $-1.924212(-2)$ & $1.924212(-2)$ \\
-0.2 & $-9.036828(-2)$ & $-6.454877(-2)$ & $-3.872926(-2)$ & $-1.290975(-2)$ & $1.290975(-2)$ \\
-0.1 & $-4.697245(-2)$ & $-3.355175(-2)$ & $-2.013105(-2)$ & $-6.710350(-3)$ & $6.710350(-3)$ \\
0.0 & $-3.641493(-3)$ & $-2.601067(-3)$ & $-1.560640(-3)$ & $-5.202134(-4)$ & $5.202134(-4)$ \\
0.1 & $4.033846(-2)$ & $2.881319(-2)$ & $1.728791(-2)$ & $5.762638(-3)$ & $-5.762638(-3)$ \\
0.2 & $8.579828(-2)$ & $6.128449(-2)$ & $3.677069(-2)$ & $1.225689(-2)$ & $-1.225689(-2)$ \\
0.3 & $1.340435(-1)$ & $9.574539(-2)$ & $5.744723(-2)$ & $1.914907(-2)$ & $-1.914907(-2)$ \\
0.4 & $1.879338(-1)$ & $1.342384(-1)$ & $8.054309(-2)$ & $2.684769(-2)$ & $-2.684769(-2)$ \\
0.5 & $2.634701(-1)$ & $1.881929(-1)$ & $1.129157(-1)$ & $3.763859(-2)$ & $-3.763859(-2)$ \\
\hline \hline
\end{tabular}

Table 9. The heat-transfer problem: density perturbation $N(\tau)$, Gross-Jackson model, $\varepsilon=\varepsilon_{t}$, $2 a=1.0, \delta_{1}=9.0, \delta_{2}=1.0$

\begin{tabular}{cccccc}
\hline \hline & $\alpha_{1}=0.5$ & $\alpha_{1}=0.5$ & $\alpha_{1}=0.5$ & $\alpha_{1}=0.5$ & \multicolumn{1}{l}{$\alpha_{1}=0.5$} \\
$\tau$ & $\alpha_{2}=0.1$ & $\alpha_{2}=0.3$ & $\alpha_{2}=0.5$ & $\alpha_{2}=0.7$ & $\alpha_{2}=1.0$ \\
\hline-0.5 & $-3.059617(-1)$ & $-7.049584(-1)$ & $-9.525032(-1)$ & -1.120026 & -1.288053 \\
-0.4 & $-2.038719(-1)$ & $-4.673100(-1)$ & $-6.281692(-1)$ & $-7.348936(-1)$ & $-8.387667(-1)$ \\
-0.3 & $-1.449270(-1)$ & $-3.304296(-1)$ & $-4.418011(-1)$ & $-5.140980(-1)$ & $-5.820501(-1)$ \\
-0.2 & $-9.494907(-2)$ & $-2.146986(-1)$ & $-2.846630(-1)$ & $-3.284339(-1)$ & $-3.670298(-1)$ \\
-0.1 & $-4.857717(-2)$ & $-1.076907(-1)$ & $-1.398655(-1)$ & $-1.579263(-1)$ & $-1.705392(-1)$ \\
0.0 & $-3.389050(-3)$ & $-3.873925(-3)$ & 0.000000 & $6.059666(-3)$ & $1.722072(-2)$ \\
0.1 & $4.232772(-2)$ & $1.005487(-1)$ & $1.398655(-1)$ & $1.690931(-1)$ & $2.022638(-1)$ \\
0.2 & $9.042127(-2)$ & $2.095291(-1)$ & $2.846630(-1)$ & $3.365017(-1)$ & $3.899190(-1)$ \\
0.3 & $1.438400(-1)$ & $3.291989(-1)$ & $4.418011(-1)$ & $5.159868(-1)$ & $5.873428(-1)$ \\
0.4 & $2.095463(-1)$ & $4.738062(-1)$ & $6.281692(-1)$ & $7.247019(-1)$ & $8.097408(-1)$ \\
0.5 & $3.337644(-1)$ & $7.365653(-1)$ & $9.525032(-1)$ & 1.071114 & 1.150140 \\
\hline \hline
\end{tabular}


Table 10. The heat-transfer problem: density perturbation $N(\tau)$, MRS model, $\varepsilon=\varepsilon_{t}, 2 a=1.0$,

\begin{tabular}{rccccc}
\multicolumn{5}{c}{$\delta_{1}=9.0, \delta_{2}=1.0$} \\
\multicolumn{1}{c}{$\alpha_{1}=0.5$} & $\alpha_{1}=0.5$ & $\alpha_{1}=0.5$ & $\alpha_{1}=0.5$ & $\alpha_{1}=0.5$ \\
\hline-0.5 & $-2.683578(-1)$ & $-6.248364(-1)$ & $-8.484871(-1)$ & -1.000019 & -1.151167 \\
-0.4 & $-1.986421(-1)$ & $-4.612411(-1)$ & $-6.246805(-1)$ & $-7.343725(-1)$ & $-8.423028(-1)$ \\
-0.3 & $-1.454753(-1)$ & $-3.366964(-1)$ & $-4.545762(-1)$ & $-5.327792(-1)$ & $-6.084172(-1)$ \\
-0.2 & $-9.635783(-2)$ & $-2.218816(-1)$ & $-2.980764(-1)$ & $-3.476625(-1)$ & $-3.942233(-1)$ \\
-0.1 & $-4.894297(-2)$ & $-1.113299(-1)$ & $-1.477543(-1)$ & $-1.702661(-1)$ & $-1.896364(-1)$ \\
0.0 & $-2.051300(-3)$ & $-2.339855(-3)$ & 0.000000 & $3.598036(-3)$ & $1.005335(-2)$ \\
0.1 & $4.520680(-2)$ & $1.070683(-1)$ & $1.477543(-1)$ & $1.768191(-1)$ & $2.079455(-1)$ \\
0.2 & $9.378624(-2)$ & $2.189485(-1)$ & $2.980764(-1)$ & $3.521715(-1)$ & $4.068182(-1)$ \\
0.3 & $1.451122(-1)$ & $3.362830(-1)$ & $4.545762(-1)$ & $5.334116(-1)$ & $6.101757(-1)$ \\
0.4 & $2.022318(-1)$ & $4.653371(-1)$ & $6.246805(-1)$ & $7.280695(-1)$ & $8.246815(-1)$ \\
0.5 & $2.820535(-1)$ & $6.404404(-1)$ & $8.484871(-1)$ & $9.760875(-1)$ & 1.084443 \\
\hline \hline
\end{tabular}

Table 11. The heat-transfer problem: temperature perturbation $T(\tau)$, Gross-Jackson model,

\begin{tabular}{cccccc}
\multicolumn{6}{c}{$\varepsilon=\varepsilon_{t}, 2 a=1.0, \alpha_{1}=0.9, \alpha_{2}=0.1$} \\
\hline \hline & $\delta_{1}=8.0$ & $\delta_{1}=8.0$ & $\delta_{1}=8.0$ & $\delta_{1}=8.0$ & $\delta_{1}=8.0$ \\
$\tau$ & $\delta_{2}=1.0$ & $\delta_{2}=3.0$ & $\delta_{2}=5.0$ & $\delta_{2}=7.0$ & $\delta_{2}=9.0$ \\
\hline-0.5 & 7.676816 & 7.769154 & 7.861492 & 7.953830 & 8.046169 \\
-0.4 & 7.588964 & 7.706402 & 7.823841 & 7.941280 & 8.058719 \\
-0.3 & 7.531664 & 7.665474 & 7.799284 & 7.933094 & 8.066905 \\
-0.2 & 7.481060 & 7.629329 & 7.777597 & 7.925865 & 8.074134 \\
-0.1 & 7.432940 & 7.594957 & 7.756974 & 7.918991 & 8.081008 \\
0.0 & 7.385170 & 7.560836 & 7.736501 & 7.912167 & 8.087832 \\
0.1 & 7.336044 & 7.525746 & 7.715447 & 7.905149 & 8.094850 \\
0.2 & 7.283561 & 7.488258 & 7.692955 & 7.897651 & 8.102348 \\
0.3 & 7.224457 & 7.446040 & 7.667624 & 7.889208 & 8.110791 \\
0.4 & 7.151186 & 7.393704 & 7.636222 & 7.878740 & 8.121259 \\
0.5 & 7.016785 & 7.297703 & 7.578622 & 7.859540 & 8.140459 \\
\hline
\end{tabular}

Table 12. The heat-transfer problem: temperature perturbation $T(\tau)$, MRS model, $\varepsilon=\varepsilon_{t}$,

\begin{tabular}{cccccc}
\multicolumn{6}{c}{$2 a=1.0, \alpha_{1}=0.9, \alpha_{2}=0.1$} \\
\hline \hline & $\delta_{1}=8.0$ & $\delta_{1}=8.0$ & $\delta_{1}=8.0$ & $\delta_{1}=8.0$ & $\delta_{1}=8.0$ \\
$\delta_{2}=1.0$ & $\delta_{2}=3.0$ & $\delta_{2}=5.0$ & $\delta_{2}=7.0$ & $\delta_{2}=9.0$ \\
\hline-0.5 & 7.650653 & 7.750466 & 7.850280 & 7.950093 & 8.049906 \\
-0.4 & 7.588271 & 7.705907 & 7.823544 & 7.941181 & 8.058818 \\
-0.3 & 7.537947 & 7.669962 & 7.801977 & 7.933992 & 8.066007 \\
-0.2 & 7.490621 & 7.636158 & 7.781694 & 7.927231 & 8.072768 \\
-0.1 & 7.444425 & 7.603161 & 7.761896 & 7.920632 & 8.079367 \\
0.0 & 7.398334 & 7.570238 & 7.742143 & 7.914047 & 8.085952 \\
0.1 & 7.351502 & 7.536787 & 7.722072 & 7.907357 & 8.092642 \\
0.2 & 7.302956 & 7.502111 & 7.701267 & 7.900422 & 8.099577 \\
0.3 & 7.251200 & 7.465142 & 7.679085 & 7.893028 & 8.106971 \\
0.4 & 7.193044 & 7.423603 & 7.654162 & 7.884720 & 8.115279 \\
0.5 & 7.111541 & 7.365386 & 7.619232 & 7.873077 & 8.126922 \\
\hline \hline
\end{tabular}


Table 13. The heat-transfer problem: temperature perturbation $T(\tau)$, Gross-Jackson model,

\begin{tabular}{cccccc}
\multicolumn{6}{c}{$\varepsilon=\varepsilon_{t}, 2 a=1.0, \delta_{1}=9.0, \delta_{2}=1.0$} \\
\hline \hline & $\alpha_{1}=0.5$ & $\alpha_{1}=0.5$ & $\alpha_{1}=0.5$ & $\alpha_{1}=0.5$ & $\alpha_{1}=0.5$ \\
$\tau$ & $\alpha_{2}=0.1$ & $\alpha_{2}=0.3$ & $\alpha_{2}=0.5$ & $\alpha_{2}=0.7$ & $\alpha_{2}=1.0$ \\
\hline-0.5 & 8.085923 & 6.871613 & 6.094378 & 5.548556 & 4.971458 \\
-0.4 & 7.970051 & 6.602303 & 5.727363 & 5.113321 & 4.464645 \\
-0.3 & 7.901202 & 6.442828 & 5.510740 & 4.857237 & 4.167778 \\
-0.2 & 7.842827 & 6.308135 & 5.328462 & 4.642532 & 3.920175 \\
-0.1 & 7.788759 & 6.183960 & 5.161178 & 4.446359 & 3.695399 \\
0.0 & 7.736073 & 6.063644 & 5.000000 & 4.258388 & 3.481767 \\
0.1 & 7.682635 & 5.942475 & 4.838821 & 4.071739 & 3.271873 \\
0.2 & 7.626149 & 5.815551 & 4.671537 & 3.879825 & 3.059121 \\
0.3 & 7.563062 & 5.675504 & 4.489259 & 3.673408 & 2.834911 \\
0.4 & 7.485353 & 5.505971 & 4.272636 & 3.432867 & 2.581857 \\
0.5 & 7.343476 & 5.207272 & 3.905621 & 3.042625 & 2.200993 \\
\hline \hline
\end{tabular}

Table 14. The heat-transfer problem: temperature perturbation $T(\tau)$, MRS model, $\varepsilon=\varepsilon_{t}$, $2 a=1.0, \delta_{1}=9.0, \delta_{2}=1.0$

\begin{tabular}{cccccc}
\hline \hline & $\alpha_{1}=0.5$ & $\alpha_{1}=0.5$ & $\alpha_{1}=0.5$ & $\alpha_{1}=0.5$ & $\alpha_{1}=0.5$ \\
$\tau$ & $\alpha_{2}=0.1$ & $\alpha_{2}=0.3$ & $\alpha_{2}=0.5$ & $\alpha_{2}=0.7$ & $\alpha_{2}=1.0$ \\
\hline-0.5 & 8.041472 & 6.746370 & 5.911396 & 5.327872 & 4.720662 \\
-0.4 & 7.965941 & 6.569041 & 5.668697 & 5.039693 & 4.385443 \\
-0.3 & 7.908620 & 6.434761 & 5.485304 & 4.822373 & 4.133378 \\
-0.2 & 7.855976 & 6.311734 & 5.317669 & 4.624171 & 3.904224 \\
-0.1 & 7.805337 & 6.193727 & 5.157310 & 4.435070 & 3.686425 \\
0.0 & 7.755331 & 6.077584 & 5.000000 & 4.250155 & 3.474439 \\
0.1 & 7.704913 & 5.960967 & 4.842689 & 4.065982 & 3.264551 \\
0.2 & 7.652968 & 5.841457 & 4.682330 & 3.879231 & 3.053404 \\
0.3 & 7.597863 & 5.715601 & 4.514695 & 3.685455 & 2.836786 \\
0.4 & 7.536202 & 5.576328 & 4.331302 & 3.475945 & 2.606840 \\
0.5 & 7.450079 & 5.386832 & 4.088603 & 3.206744 & 2.325234 \\
\hline \hline
\end{tabular}

Table 15. The heat-transfer problem: heat flow $Q_{x}, \varepsilon=\varepsilon_{t}, 2 a=5.0, \delta_{1}=8.0$ and $\delta_{2}=1.0$

\begin{tabular}{ccllll}
\hline \hline$\alpha_{1}$ & $\alpha_{2}$ & \multicolumn{1}{c}{ BGK } & \multicolumn{1}{c}{ S } & \multicolumn{1}{c}{ GJ } & \multicolumn{1}{c}{ MRS } \\
\hline 0.7 & 0.1 & $3.045766(-1)$ & $3.042991(-1)$ & $3.041093(-1)$ & $3.051667(-1)$ \\
0.7 & 0.3 & $6.289031(-1)$ & $6.278496(-1)$ & $6.271531(-1)$ & $6.311507(-1)$ \\
0.7 & 0.5 & $8.015793(-1)$ & $8.000725(-1)$ & $7.991031(-1)$ & $8.048080(-1)$ \\
0.7 & 0.7 & $9.106524(-1)$ & $9.089658(-1)$ & $9.079011(-1)$ & $9.142955(-1)$ \\
0.7 & 0.9 & $9.869966(-1)$ & $9.853123(-1)$ & $9.842579(-1)$ & $9.906865(-1)$ \\
0.7 & 1.0 & 1.017474 & 1.015839 & 1.014815 & 1.021092 \\
0.9 & 0.1 & $3.126463(-1)$ & $3.123956(-1)$ & $3.122207(-1)$ & $3.131954(-1)$ \\
0.9 & 0.3 & $6.643369(-1)$ & $6.633313(-1)$ & $6.626591(-1)$ & $6.665355(-1)$ \\
0.9 & 0.5 & $8.600947(-1)$ & $8.586151(-1)$ & $8.576613(-1)$ & $8.633282(-1)$ \\
0.9 & 0.7 & $9.869966(-1)$ & $9.853123(-1)$ & $9.842579(-1)$ & $9.906865(-1)$ \\
0.9 & 0.9 & 1.077387 & 1.075687 & 1.074644 & 1.081139 \\
0.9 & 1.0 & 1.113845 & 1.112190 & 1.111179 & 1.117524 \\
1.0 & 1.0 & 1.152879 & 1.151264 & 1.150288 & 1.156482 \\
\hline \hline
\end{tabular}


Table 16. The heat-transfer problem: heat flow $Q_{x}, \varepsilon=\varepsilon_{t}, 2 a=2.0, \delta_{1}=8.0$ and $\delta_{2}=1.0$

\begin{tabular}{ccllll}
\hline \hline$\alpha_{1}$ & $\alpha_{2}$ & \multicolumn{1}{c}{ BGK } & \multicolumn{1}{c}{ S } & \multicolumn{1}{c}{ GJ } & \multicolumn{1}{c}{ MRS } \\
\hline 0.7 & 0.1 & $3.418737(-1)$ & $3.409296(-1)$ & $3.401526(-1)$ & $3.429983(-1)$ \\
0.7 & 0.3 & $8.107691(-1)$ & $8.059813(-1)$ & $8.021232(-1)$ & $8.165441(-1)$ \\
0.7 & 0.5 & 1.120644 & 1.112450 & 1.105963 & 1.130642 \\
0.7 & 0.7 & 1.343425 & 1.332971 & 1.324829 & 1.356348 \\
0.7 & 0.9 & 1.513230 & 1.501594 & 1.492674 & 1.527854 \\
0.7 & 1.0 & 1.584328 & 1.572446 & 1.563413 & 1.599414 \\
0.9 & 0.1 & $3.518597(-1)$ & $3.509624(-1)$ & $3.502341(-1)$ & $3.529537(-1)$ \\
0.9 & 0.3 & $8.694020(-1)$ & $8.644953(-1)$ & $8.605981(-1)$ & $8.754478(-1)$ \\
0.9 & 0.5 & 1.236106 & 1.227286 & 1.220409 & 1.247075 \\
0.9 & 0.7 & 1.513230 & 1.501594 & 1.492674 & 1.527854 \\
0.9 & 0.9 & 1.732709 & 1.719453 & 1.709460 & 1.749592 \\
0.9 & 1.0 & 1.826868 & 1.813217 & 1.803014 & 1.844401 \\
1.0 & 1.0 & 1.932021 & 1.917923 & 1.907476 & 1.950265 \\
\hline \hline
\end{tabular}

Table 17. The heat-transfer problem: heat flow $Q_{x}, \varepsilon=\varepsilon_{t}, 2 a=0.2, \delta_{1}=8.0$ and $\delta_{2}=1.0$

\begin{tabular}{ccllll}
\hline \hline$\alpha_{1}$ & $\alpha_{2}$ & \multicolumn{1}{c}{ BGK } & \multicolumn{1}{c}{ S } & \multicolumn{1}{c}{ GJ } & \multicolumn{1}{c}{ MRS } \\
\hline 0.7 & 0.1 & $3.736442(-1)$ & $3.729568(-1)$ & $3.720839(-1)$ & $3.741735(-1)$ \\
0.7 & 0.3 & 1.012290 & 1.007531 & 1.001578 & 1.016002 \\
0.7 & 0.5 & 1.538824 & 1.528437 & 1.515622 & 1.547023 \\
0.7 & 0.7 & 1.981056 & 1.964782 & 1.944964 & 1.994050 \\
0.7 & 0.9 & 2.358233 & 2.336420 & 2.310191 & 2.375852 \\
0.7 & 1.0 & 2.526885 & 2.502523 & 2.473413 & 2.546675 \\
0.9 & 0.1 & $3.852405(-1)$ & $3.845305(-1)$ & $3.836360(-1)$ & $3.857910(-1)$ \\
0.9 & 0.3 & 1.102234 & 1.096792 & 1.090048 & 1.106513 \\
0.9 & 0.5 & 1.756921 & 1.743980 & 1.728185 & 1.767236 \\
0.9 & 0.7 & 2.358233 & 2.336420 & 2.310191 & 2.375852 \\
0.9 & 0.9 & 2.913413 & 2.882291 & 2.845413 & 2.938885 \\
0.9 & 1.0 & 3.175602 & 3.139866 & 3.097831 & 3.205046 \\
1.0 & 1.0 & 3.489892 & 3.448330 & 3.399827 & 3.524382 \\
\hline \hline
\end{tabular}


Table 18. The heat-transfer problem: heat flow $Q_{x}$, Gross-Jackson model, $\varepsilon=\varepsilon_{t}$ and $2 a=1.0$

\begin{tabular}{ccccccc}
\hline \hline & & \multicolumn{1}{c}{$\delta_{1}=8.0$} & \multicolumn{1}{c}{$\delta_{1}=8.0$} & $\delta_{1}=8.0$ & $\delta_{1}=8.0$ & $\delta_{1}=8.0$ \\
$\alpha_{1}$ & $\alpha_{2}$ & \multicolumn{1}{c}{$\delta_{2}=0.0$} & \multicolumn{1}{c}{$\delta_{2}=1.0$} & \multicolumn{1}{c}{$\delta_{2}=3.0$} & $\delta_{2}=5.0$ & $\delta_{2}=7.0$ \\
\hline 0.7 & 0.1 & $4.061911(-1)$ & $3.554172(-1)$ & $2.538694(-1)$ & $1.523216(-1)$ & $5.077389(-2)$ \\
0.7 & 0.3 & 1.018915 & $8.915513(-1)$ & $6.368223(-1)$ & $3.820934(-1)$ & $1.273644(-1)$ \\
0.7 & 0.5 & 1.464610 & 1.281533 & $9.153812(-1)$ & $5.492287(-1)$ & $1.830762(-1)$ \\
0.7 & 0.7 & 1.808080 & 1.582070 & 1.130050 & $6.780300(-1)$ & $2.260100(-1)$ \\
0.7 & 0.9 & 2.084291 & 1.823755 & 1.302682 & $7.816094(-1)$ & $2.605364(-1)$ \\
0.7 & 1.0 & 2.203941 & 1.928449 & 1.377463 & $8.264782(-1)$ & $2.754927(-1)$ \\
0.9 & 0.1 & $4.185768(-1)$ & $3.662547(-1)$ & $2.616105(-1)$ & $1.569663(-1)$ & $5.232210(-2)$ \\
0.9 & 0.3 & 1.100785 & $9.631872(-1)$ & $6.879908(-1)$ & $4.127945(-1)$ & $1.375981(-1)$ \\
0.9 & 0.5 & 1.640329 & 1.435288 & 1.025205 & $6.151235(-1)$ & $2.050411(-1)$ \\
0.9 & 0.7 & 2.084291 & 1.823755 & 1.302682 & $7.816094(-1)$ & $2.605364(-1)$ \\
0.9 & 0.9 & 2.461040 & 2.153410 & 1.538150 & $9.228901(-1)$ & $3.076300(-1)$ \\
0.9 & 1.0 & 2.630137 & 2.301370 & 1.643836 & $9.863017(-1)$ & $3.287672(-1)$ \\
1.0 & 1.0 & 2.824499 & 2.471437 & 1.765312 & 1.059187 & $3.530624(-1)$ \\
\hline \hline
\end{tabular}

Table 19. The heat-transfer problem: heat flow $Q_{x}$, MRS model, $\varepsilon=\varepsilon_{t}$ and $2 a=1.0$

\begin{tabular}{ccccccc}
\hline \hline & & \multicolumn{1}{c}{$\delta_{1}=8.0$} & \multicolumn{1}{c}{$\delta_{1}=8.0$} & $\delta_{1}=8.0$ & $\delta_{1}=8.0$ & $\delta_{1}=8.0$ \\
$\alpha_{1}$ & $\alpha_{2}$ & \multicolumn{1}{c}{$\delta_{2}=0.0$} & \multicolumn{1}{c}{$\delta_{2}=1.0$} & \multicolumn{1}{c}{$\delta_{2}=3.0$} & $\delta_{2}=5.0$ & $\delta_{2}=7.0$ \\
\hline 0.7 & 0.1 & $4.101947(-1)$ & $3.589203(-1)$ & $2.563717(-1)$ & $1.538230(-1)$ & $5.127434(-2)$ \\
0.7 & 0.3 & 1.042264 & $9.119815(-1)$ & $6.514153(-1)$ & $3.908492(-1)$ & $1.302830(-1)$ \\
0.7 & 0.5 & 1.508874 & 1.320265 & $9.430467(-1)$ & $5.658280(-1)$ & $1.886093(-1)$ \\
0.7 & 0.7 & 1.869448 & 1.635767 & 1.168405 & $7.010432(-1)$ & $2.336810(-1)$ \\
0.7 & 0.9 & 2.157865 & 1.888132 & 1.348666 & $8.091997(-1)$ & $2.697332(-1)$ \\
0.7 & 1.0 & 2.281828 & 1.996599 & 1.426142 & $8.556855(-1)$ & $2.852285(-1)$ \\
0.9 & 0.1 & $4.225197(-1)$ & $3.697047(-1)$ & $2.640748(-1)$ & $1.584448(-1)$ & $5.281496(-2)$ \\
0.9 & 0.3 & 1.125847 & $9.851167(-1)$ & $7.036548(-1)$ & $4.221928(-1)$ & $1.407309(-1)$ \\
0.9 & 0.5 & 1.690939 & 1.479572 & 1.056837 & $6.341023(-1)$ & $2.113674(-1)$ \\
0.9 & 0.7 & 2.157865 & 1.888132 & 1.348666 & $8.091997(-1)$ & $2.697332(-1)$ \\
0.9 & 0.9 & 2.552455 & 2.233398 & 1.595284 & $9.571708(-1)$ & $3.190569(-1)$ \\
0.9 & 1.0 & 2.728294 & 2.387257 & 1.705183 & 1.023110 & $3.410367(-1)$ \\
1.0 & 1.0 & 2.930488 & 2.564177 & 1.831555 & 1.098933 & $3.663110(-1)$ \\
\hline \hline
\end{tabular}




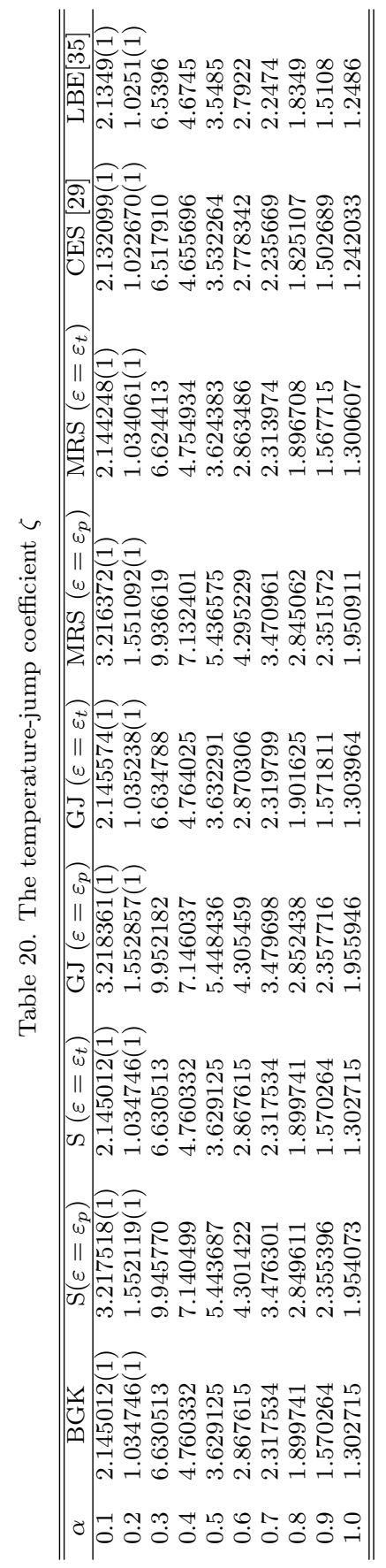




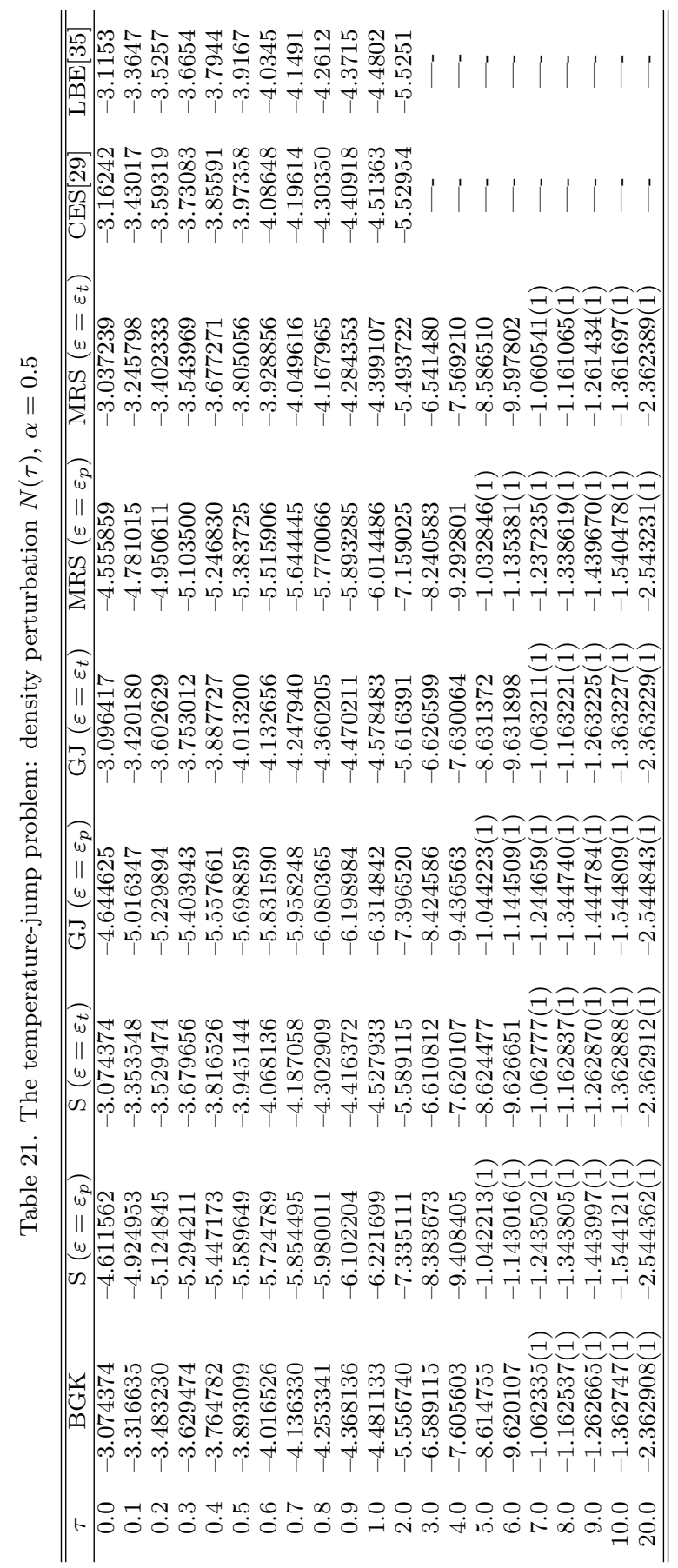




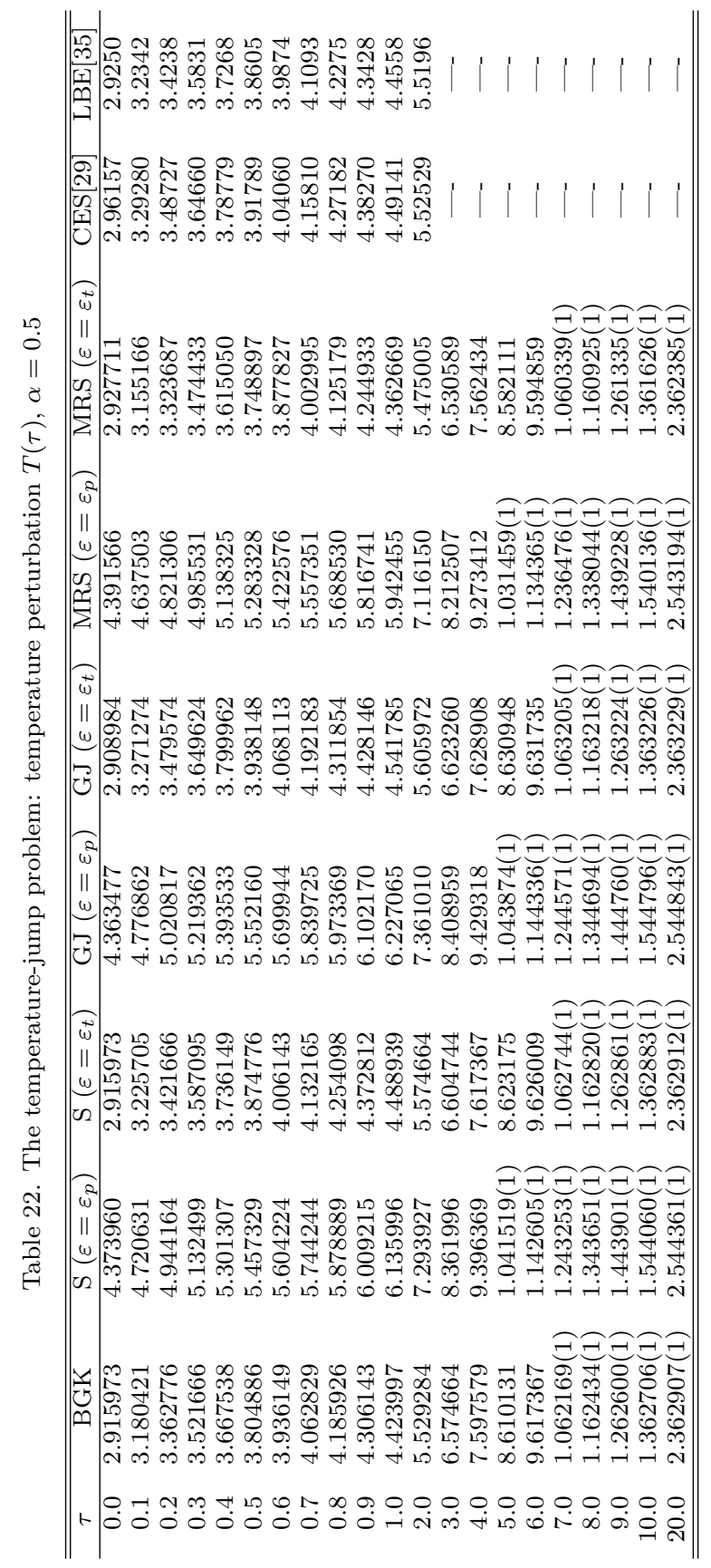


Table 23. The temperature-jump problem: density perturbation $N(\tau)$, Gross-Jackson model

\begin{tabular}{rcccrcc}
\hline \hline \multicolumn{4}{c}{$\alpha=0.1$} & \multicolumn{2}{c}{$\alpha=0.3$} & \multicolumn{2}{c}{$\alpha=1.0$} \\
$\tau$ & $\varepsilon=\varepsilon_{p}$ & $\varepsilon=\varepsilon_{t}$ & $\varepsilon=\varepsilon_{p}$ & $\varepsilon=\varepsilon_{t}$ & $\varepsilon=\varepsilon_{p}$ & $\varepsilon=\varepsilon_{t}$ \\
\hline 0.0 & $-3.112351(1)$ & $-2.074901(1)$ & -9.022763 & -6.015175 & -1.445581 & $-9.637207(-1)$ \\
0.1 & $-3.158919(1)$ & $-2.114919(1)$ & -9.440136 & -6.376117 & -1.713814 & -1.202815 \\
0.2 & $-3.183919(1)$ & $-2.135760(1)$ & -9.671538 & -6.571312 & -1.885744 & -1.355340 \\
0.3 & $-3.203641(1)$ & $-2.152345(1)$ & -9.856968 & -6.729306 & -2.033010 & -1.487678 \\
0.4 & $-3.220666(1)$ & $-2.166865(1)$ & $-1.001882(1)$ & -6.869193 & -2.167461 & -1.610057 \\
0.5 & $-3.236038(1)$ & $-2.180174(1)$ & $-1.016619(1)$ & -6.998426 & -2.293971 & -1.726528 \\
0.6 & $-3.250298(1)$ & $-2.192697(1)$ & $-1.030379(1)$ & -7.120733 & -2.415086 & -1.839135 \\
0.7 & $-3.263762(1)$ & $-2.204676(1)$ & $-1.043438(1)$ & -7.238247 & -2.532316 & -1.949055 \\
0.8 & $-3.276632(1)$ & $-2.216263(1)$ & $-1.055975(1)$ & -7.352294 & -2.646634 & -2.057024 \\
0.9 & $-3.289045(1)$ & $-2.227556(1)$ & $-1.068110(1)$ & -7.463749 & -2.758703 & -2.163531 \\
1.0 & $-3.301099(1)$ & $-2.238625(1)$ & $-1.079927(1)$ & -7.573217 & -2.868993 & -2.268914 \\
2.0 & $-3.411656(1)$ & $-2.343519(1)$ & $-1.189277(1)$ & -8.616588 & -3.922127 & -3.293612 \\
3.0 & $-3.515279(1)$ & $-2.444838(1)$ & $-1.292488(1)$ & -9.628270 & -4.940418 & -4.300261 \\
4.0 & $-3.616826(1)$ & $-2.545286(1)$ & $-1.393858(1)$ & $-1.063223(1)$ & -5.948221 & -5.302516 \\
5.0 & $-3.717559(1)$ & $-2.645455(1)$ & $-1.494508(1)$ & $-1.163373(1)$ & -6.951915 & -6.303367 \\
6.0 & $-3.817929(1)$ & $-2.745523(1)$ & $-1.594835(1)$ & $-1.263433(1)$ & -7.953775 & -7.303709 \\
7.0 & $-3.918123(1)$ & $-2.845552(1)$ & $-1.695007(1)$ & $-1.363459(1)$ & -8.954749 & -8.303852 \\
8.0 & $-4.018227(1)$ & $-2.945564(1)$ & $-1.795099(1)$ & $-1.463469(1)$ & -9.955274 & -9.303913 \\
9.0 & $-4.118285(1)$ & $-3.045569(1)$ & $-1.895150(1)$ & $-1.563474(1)$ & $-1.095556(1)$ & $-1.030394(1)$ \\
10.0 & $-4.218317(1)$ & $-3.145572(1)$ & $-1.995179(1)$ & $-1.663476(1)$ & $-1.195572(1)$ & $-1.130395(1)$ \\
20.0 & $-5.218361(1)$ & $-4.145574(1)$ & $-2.995217(1)$ & $-2.663478(1)$ & $-2.195594(1)$ & $-2.130396(1)$ \\
\hline \hline
\end{tabular}

Table 24. The temperature-jump problem: density perturbation $N(\tau)$, MRS model

\begin{tabular}{rrrrrrr}
\hline \hline \multicolumn{6}{c}{$\alpha=0.1$} & \multicolumn{2}{c}{$\alpha=0.3$} & \multicolumn{2}{c}{$\alpha=1.0$} \\
$\tau$ & $\varepsilon=\varepsilon_{p}$ & $\varepsilon=\varepsilon_{t}$ & $\varepsilon=\varepsilon_{p}$ & $\varepsilon=\varepsilon_{t}$ & $\varepsilon=\varepsilon_{p}$ & $\varepsilon=\varepsilon_{t}$ \\
\hline 0.0 & $-3.097660(1)$ & $-2.065107(1)$ & -8.907059 & -5.938039 & -1.407291 & $-9.381942(-1)$ \\
0.1 & $-3.125265(1)$ & $-2.090326(1)$ & -9.156580 & -6.167509 & -1.580157 & -1.101692 \\
0.2 & $-3.144892(1)$ & $-2.108102(1)$ & -9.339020 & -6.334287 & -1.721613 & -1.235629 \\
0.3 & $-3.162154(1)$ & $-2.123783(1)$ & -9.501431 & -6.483260 & -1.853443 & -1.360904 \\
0.4 & $-3.178071(1)$ & $-2.138300(1)$ & -9.652423 & -6.622316 & -1.979711 & -1.481276 \\
0.5 & $-3.193089(1)$ & $-2.152052(1)$ & -9.795749 & -6.754829 & -2.102204 & -1.598376 \\
0.6 & $-3.207450(1)$ & $-2.165255(1)$ & -9.933474 & -6.882630 & -2.221915 & -1.713093 \\
0.7 & $-3.221306(1)$ & $-2.178040(1)$ & $-1.006688(1)$ & -7.006841 & -2.339464 & -1.825980 \\
0.8 & $-3.234760(1)$ & $-2.190495(1)$ & $-1.019683(1)$ & -7.128215 & -2.455271 & -1.937410 \\
0.9 & $-3.247882(1)$ & $-2.202684(1)$ & $-1.032394(1)$ & -7.247281 & -2.569639 & -2.047647 \\
1.0 & $-3.260728(1)$ & $-2.214650(1)$ & $-1.044867(1)$ & -7.364432 & -2.682793 & -2.156887 \\
2.0 & $-3.379993(1)$ & $-2.327178(1)$ & $-1.161669(1)$ & -8.474049 & -3.773301 & -3.216648 \\
3.0 & $-3.490767(1)$ & $-2.433450(1)$ & $-1.271107(1)$ & -9.529153 & -4.824972 & -4.247132 \\
4.0 & $-3.597630(1)$ & $-2.537076(1)$ & $-1.377134(1)$ & $-1.056107(1)$ & -5.858272 & -5.264932 \\
5.0 & $-3.702301(1)$ & $-2.639332(1)$ & $-1.481243(1)$ & $-1.158096(1)$ & -6.881110 & -6.276076 \\
6.0 & $-3.805614(1)$ & $-2.740801(1)$ & $-1.584161(1)$ & $-1.259393(1)$ & -7.897398 & -7.283368 \\
7.0 & $-3.908032(1)$ & $-2.841790(1)$ & $-1.686293(1)$ & $-1.360266(1)$ & -8.909336 & -8.288289 \\
8.0 & $-4.009835(1)$ & $-2.942471(1)$ & $-1.787884(1)$ & $-1.460868(1)$ & -9.918265 & -9.291688 \\
9.0 & $-4.111202(1)$ & $-3.042949(1)$ & $-1.889090(1)$ & $-1.561291(1)$ & $-1.092505(1)$ & $-1.029407(1)$ \\
10.0 & $-4.212252(1)$ & $-3.143289(1)$ & $-1.990017(1)$ & $-1.661592(1)$ & $-1.193027(1)$ & $-1.129578(1)$ \\
20.0 & $-5.215822(1)$ & $-4.144185(1)$ & $-2.993174(1)$ & $-2.662385(1)$ & $-2.194813(1)$ & $-2.130028(1)$ \\
\hline \hline
\end{tabular}


Table 25. The temperature-jump problem: temperature perturbation $T(\tau)$, Gross-Jackson

\begin{tabular}{|c|c|c|c|c|c|c|}
\hline \multirow[b]{2}{*}{$\tau$} & \multicolumn{2}{|c|}{$\alpha=0.1$} & \multicolumn{2}{|c|}{$\alpha=0.3$} & \multicolumn{2}{|c|}{$\alpha=1.0$} \\
\hline & $\varepsilon=\varepsilon_{p}$ & $\varepsilon=\varepsilon_{t}$ & $\varepsilon=\varepsilon_{p}$ & $\varepsilon=\varepsilon_{t}$ & $\varepsilon=\varepsilon_{p}$ & $\varepsilon=\varepsilon_{t}$ \\
\hline 0.0 & $3.073530(1)$ & $2.049020(1)$ & 8.690296 & 5.793531 & 1.276168 & $8.507787(-1)$ \\
\hline 0.1 & $3.126150(1)$ & $2.094594(1)$ & 9.158200 & 6.201029 & 1.567967 & 1.111862 \\
\hline 0.2 & $3.155439(1)$ & $2.119052(1)$ & 9.425946 & 6.426995 & 1.757731 & 1.279706 \\
\hline 0.3 & $3.178578(1)$ & $2.138357(1)$ & 9.640492 & 6.608272 & 1.919559 & 1.423854 \\
\hline 0.4 & $3.198436(1)$ & $2.155025(1)$ & 9.826574 & 6.766592 & 2.066274 & 1.555695 \\
\hline 0.5 & $3.216211(1)$ & $2.170072(1)$ & 9.994541 & 6.910787 & 2.203301 & 1.679912 \\
\hline 0.6 & $3.232537(1)$ & $2.184026(1)$ & $1.014988(1)$ & 7.045439 & 2.333543 & 1.798953 \\
\hline 0.7 & $3.247797(1)$ & $2.197198(1)$ & $1.029593(1)$ & 7.173256 & 2.458763 & 1.914271 \\
\hline 0.8 & $3.262240(1)$ & $2.209788(1)$ & $1.043485(1)$ & 7.295978 & 2.580123 & 2.026806 \\
\hline 0.9 & $3.276039(1)$ & $2.221931(1)$ & $1.056815(1)$ & 7.414789 & 2.698429 & 2.137200 \\
\hline 1.0 & $3.289321(1)$ & $2.233724(1)$ & $1.069693(1)$ & 7.530529 & 2.814269 & 2.245909 \\
\hline 2.0 & $3.406930(1)$ & $2.342139(1)$ & $1.185153(1)$ & 8.604520 & 3.899775 & 3.287015 \\
\hline 3.0 & $3.513209(1)$ & $2.444398(1)$ & $1.290678(1)$ & 9.624412 & 4.930523 & 4.298135 \\
\hline 4.0 & $3.615870(1)$ & $2.545134(1)$ & $1.393021(1)$ & $1.063090(1)$ & 5.943614 & 5.301777 \\
\hline 5.0 & $3.717100(1)$ & $2.645400(1)$ & $1.494105(1)$ & $1.163324(1)$ & 6.949688 & 6.303095 \\
\hline 6.0 & $3.817702(1)$ & $2.745502(1)$ & $1.594635(1)$ & $1.263415(1)$ & 7.952666 & 7.303604 \\
\hline 7.0 & $3.918007(1)$ & $2.845543(1)$ & $1.694905(1)$ & $1.363451(1)$ & 8.954183 & 8.303810 \\
\hline 8.0 & $4.018167(1)$ & $2.945560(1)$ & $1.795046(1)$ & $1.463466(1)$ & 9.954978 & 9.303896 \\
\hline 9.0 & $4.118253(1)$ & $3.045568(1)$ & $1.895122(1)$ & $1.563473(1)$ & $1.095540(1)$ & $1.030393(1)$ \\
\hline 10.0 & $4.218300(1)$ & $3.145571(1)$ & $1.995164(1)$ & $1.663476(1)$ & $1.195564(1)$ & $1.130395(1)$ \\
\hline 20.0 & $5.218361(1)$ & $4.145574(1)$ & $2.995217(1)$ & $2.663478(1)$ & $2.195594(1)$ & $2.130396(1)$ \\
\hline
\end{tabular}

Table 26. The temperature-jump problem: temperature perturbation $T(\tau)$, MRS model

\begin{tabular}{rrrrrrr}
\multicolumn{1}{c}{$\alpha=0.1$} & \multicolumn{2}{c}{$\alpha=0.3$} & \multicolumn{2}{c}{$\alpha=1.0$} \\
$\tau$ & $\varepsilon=\varepsilon_{p}$ & $\varepsilon=\varepsilon_{t}$ & $\varepsilon=\varepsilon_{p}$ & $\varepsilon=\varepsilon_{t}$ & $\varepsilon=\varepsilon_{p}$ & $\varepsilon=\varepsilon_{t}$ \\
\hline 0.0 & $3.078213(1)$ & $2.052142(1)$ & 8.727037 & 5.818025 & 1.288190 & $8.587937(-1)$ \\
0.1 & $3.107989(1)$ & $2.079372(1)$ & 8.998042 & 6.067194 & 1.478004 & 1.037467 \\
0.2 & $3.129215(1)$ & $2.098520(1)$ & 9.195680 & 6.246894 & 1.630311 & 1.180409 \\
0.3 & $3.147780(1)$ & $2.115264(1)$ & 9.370341 & 6.405785 & 1.770614 & 1.312387 \\
0.4 & $3.164801(1)$ & $2.130647(1)$ & 9.531625 & 6.552852 & 1.903861 & 1.438073 \\
0.5 & $3.180772(1)$ & $2.145122(1)$ & 9.683808 & 6.692031 & 2.032277 & 1.559535 \\
0.6 & $3.195970(1)$ & $2.158940(1)$ & 9.829278 & 6.825490 & 2.157110 & 1.677916 \\
0.7 & $3.210570(1)$ & $2.172257(1)$ & 9.969538 & 6.954577 & 2.279152 & 1.793933 \\
0.8 & $3.224688(1)$ & $2.185178(1)$ & $1.010561(1)$ & 7.080200 & 2.398943 & 1.908072 \\
0.9 & $3.238411(1)$ & $2.197776(1)$ & $1.023823(1)$ & 7.203006 & 2.516874 & 2.020678 \\
1.0 & $3.251801(1)$ & $2.210106(1)$ & $1.036795(1)$ & 7.323472 & 2.633235 & 2.132008 \\
2.0 & $3.374623(1)$ & $2.324819(1)$ & $1.156839(1)$ & 8.452900 & 3.744188 & 3.204035 \\
3.0 & $3.487229(1)$ & $2.432070(1)$ & $1.267935(1)$ & 9.516813 & 4.806053 & 4.239846 \\
4.0 & $3.595177(1)$ & $2.536214(1)$ & $1.374939(1)$ & $1.055338(1)$ & 5.845275 & 5.260420 \\
5.0 & $3.700542(1)$ & $2.638771(1)$ & $1.479671(1)$ & $1.157596(1)$ & 6.871852 & 6.273156 \\
6.0 & $3.804322(1)$ & $2.740425(1)$ & $1.583008(1)$ & $1.259058(1)$ & 7.890631 & 7.281419 \\
7.0 & $3.907065(1)$ & $2.841532(1)$ & $1.685431(1)$ & $1.360037(1)$ & 8.904292 & 8.286958 \\
8.0 & $4.009101(1)$ & $2.942291(1)$ & $1.787229(1)$ & $1.460708(1)$ & 9.914449 & 9.290762 \\
9.0 & $4.110638(1)$ & $3.042822(1)$ & $1.888588(1)$ & $1.561178(1)$ & $1.092212(1)$ & $1.029342(1)$ \\
10.0 & $4.211814(1)$ & $3.143198(1)$ & $1.989628(1)$ & $1.661511(1)$ & $1.192801(1)$ & $1.129531(1)$ \\
20.0 & $5.215774(1)$ & $4.144180(1)$ & $2.993132(1)$ & $2.662381(1)$ & $2.194789(1)$ & $2.130026(1)$ \\
\hline \hline
\end{tabular}




\section{S. Scherer}

Programa de Pós-Graduação em Engenharia Mecânica

Universidade Federal do Rio Grande do Sul

Rua Sarmento Leite, 425

90050-170 Porto Alegre, RS, Brasil

e-mail: csscherer@unisinos.br

Permanent address:

Universidade do Vale do Rio dos Sinos

Unisinos, São Leopoldo, RS, Brasil

J. F. Prolo Filho

Programa de Pós-Graduação em Matemática Aplicada

e-mail: joprolo@gmail.com

L. B. Barichello

Instituto de Matemática

Universidade Federal do Rio Grande do Sul

Av. Bento Gonçalves, 9500 - Campus do Vale

91509-900 Porto Alegre, RS, Brasil

e-mail: lbaric@ufrgs.br

(Received: September 19, 2007; revised: April 11, 2008)

Published Online First: November 26, 2008 